\title{
UNILATERAL CONTACT AND DRY FRICTION \\ IN FINITE FREEDOM DYNAMICS
}

\author{
J.J. Moreau \\ Université des Sciences et Techniques du Languedoc \\ Montpellier, France
}

\begin{abstract}
An approach to the dynamics of mechanical systems with a finite number of degrees of freedom, involving unilateral constraints, is developed. In the n-dimensional linear spaces of forces and velocities, some classical concepts of Convex Analysis are used, but no convexity assumption is made concerning the constraint inequalities. The velocity is not supposed to be a differentiable function of time, but only to have locally bounded variation, so the role of the acceleration is held by a n-dimensional measure on the considered time interval. Dynamics is then governed by measure differential inclusions, which treat possible velocity jumps on the same footing as smooth motions. Possible collisions are described as soft, thus dissipative. Friction is taken into account under a recently proposed expression of Coulomb's law. These formulations have the advantage of generating numerical algorithms of time-discretization, able to handle, in particular, the nonsmooth effects arising from unllaterality and from dry friction.
\end{abstract}




\section{INTRODUCTION}

Usual mechanisms consist of parts which, at the first level of investigation, are treated as perfectly rigid bodies. The mechanism operation rests on the fact that some of these parts may come into contact or get loose from each other, but none of them can overlap. Similarly, the parts may touch the external bodies which support the mechanism, but can never encroach upon the region of space they occupy.

In terms of the parameters $q^{1}, \ldots, q^{n}$, making an element of $\mathbb{R}^{n}$ denoted in the sequel by $q$, which are used to locate every position of the considered system, the above impenetrability properties may as a rule be expressed by a set of inequalities, say $f_{\alpha}(q) \leqslant 0, \alpha \in\{1, \ldots, \gamma\}$. Each of these inequalities corresponds to what is traditionally called a unilateral constraint. Naturally, the description of a constraint in Mechanics does not reduce to the geometric restriction it imposes to the system possible positions. Predicting the system behaviour always requires some additional information about the forces of constraint or reactions needed by the system dynamics, for the geometric conditions to be satisfied at every instant.

Constraints whose geometric effect is expressed by equalities are, in contrast, said bilateral. They are commonly realized by the conjunction of several unilateral constraints and, in practice, this may leave a residual looseness whose investigation has primary importance in some applications.

However omnipresent unilateral constraints are in machines, the place they receive in the books on Classical Mechanics is very modest. Here is the traditional approach of the situation.

Starting with a position of the system in which some of the contacts are effective (l.e. the corresponding inequalities hold as equalities) and 
with velocities compatible with the persistence of these contacts, the subsequent motion is calculated under the tentative assumption that all the said contacts do persist. The calculation is identical to what is more familiarly done in the case of bilateral constraints, with friction possibly taken into account. At every instant of the calculated motion, the respective contact forces are evaluated. As long as the direction of each of these vectors is found compatible with the unilaterality of the corresponding contact, the calculated solution is accepted (rigorously, this does not dispense from investigating also the possiblity of contact breaking, since the uniqueness of solution to an initial value problem of Dynamics is not granted in general).

But if the above calculation yields, after an instant $\tau$, a non feasible value for some of the contact forces, the tentative assumption has to be rejected from this instant onward, and other types of motions, in which some of the contacts initially present get loose, are to be tested in the same way. The number of combinations to be tried may be high, if many unllateral contacts are involved. In practical situations, such instants as $\tau$ make a finite set, but this cannot be asserted in general.

It was not before the first quarter of this century that $E$. Delassus (cf.[1] for the frictionless case) observed that, contrary to what had been formerly believed, the contacts which get loose at time $\tau$ are not necessarily those for which the calculation, performed at $t>\tau$, yields contact forces of non feasible direction. Delassus' papers seem today rather intricate; a clearer account of his arguments may be found in [2]. More recently [3][4], the same question has been revisited, by the means of elementary Convex Analysis and Quadratic Programming. 
The present lectures develop a novel approach to the dynamics of systemis involving unilateral constraints. Here are the dominent features.

$1^{\circ}$ The function $t \rightarrow q(t) \in \mathbb{R}^{n}$ describing the investigated motion on a time interval 1 , with initial instant $t_{0}$, is not a priori assumed differentiable everywhere. Instead, one supposes that a equals the time integral of a velocity function $t \rightarrow u(t) \in \mathbb{R}^{n}$ with locally bounded variation on the interval; notation: $u \in \operatorname{lbv}\left(1, \mathbb{R}^{n}\right)$. Classically, such a function $u$ may have discontinuities but, for every $t$ in the interior of 1 , the existence of the right-limit $\mathrm{u}^{+}(\mathrm{t})$ and of the left-limit $\mathrm{u}^{-}(\mathrm{t})$ is secured (see Sec. 2 below for a convention concerning the case $t=t_{0}$ ). These limits equal the respective one-side derivatives of the function $q: 1 \rightarrow \mathbb{R}^{n}$ at point $t$.

$2^{\circ}$ In view of these discontinuities, the existence of the acceleration $q^{\prime \prime}=u^{\prime}$ cannot be expected everywhere. But, with every $u \in \operatorname{lbv}\left(I, \mathbb{R}^{n}\right)$, one classically associates an $\mathbb{R}^{n}$-valued measure [5][6] on the interval 1 , called in the sequel the differential measure [7] of $u$ and denoted by du.

The function $u$ is locally absolutely continuous if and only if the vector measure du possesses a density runction, say $u_{i}^{\prime} \in \mathcal{L}^{1}{ }_{l o c}\left(1, d t ; \mathbb{R}^{n}\right)$, relative to the Lebesgue measure on the interval 1 . We denote the latter measure by $d t$; this is in fact the differential measure of the real function $t \rightarrow t$, which evidently belongs to $\operatorname{lbv}(I, \mathbb{R})$. A function $u$ of this sort may constitute a solution to a differential equation, in the classical sense of Caratheodory.

Here is another special case: suppose that, for some $\tau \in$ int 1 , one has $u^{-}(\tau) \neq u^{+}(\tau)$. Then, the $\mathbb{R}^{n}$-valued measure $d u$ possesses at point $\tau$ an atom with value $u^{+}(\tau)-u^{-}(\tau)$. This value is an element of $\mathbb{R}^{n}$ that we shall 
call the jump of $u$ at instant $\tau$.

In general, a function $u \in \operatorname{lbv}\left(1, \mathbb{R}^{n}\right)$ may be a solution to some measure differential equation, a notion about which the reader could find some information in [8].

$3^{\circ}$ Velocity functions with locally bounded variation make the setting in which we develop the Nonsmooth Dynamics of mechanical systems with a finite number of degrees of freedom. This is goverried by an extension of Lagrange equations that we introduce in Sec.7. It includes as a special case the traditional equations of the Dynamics of Percussions. Concerning the connection of this general formulation with the classical principles of Dynamics, some detalls may be found in [9].

$4^{\circ}$ The set of inequalities $f_{\alpha} \leqslant 0$ (with $f_{\alpha} \in C^{\prime}$ and $\nabla f_{\alpha} \neq 0$ ) defines in $\mathbb{R}^{n}$ the feasible region, denoted by $\Phi$ and assumed in the sequel independent of time. If a motion $t \rightarrow q(t)$ is described in the above terms and if $q(t) \in \Phi$ for every $t$, one elementarily finds (see $\operatorname{Sec} .2$ ) that $u^{+}(t)$ belongs to a certain polyhedral conic convex subset of $\mathbb{R}^{n}$, denoted by $V(q(t))$. This is the tangent cone to the region $\Phi$ at point $q(t)$, equal in particular to the whole of $\mathbb{R}^{n}$ when $q(t)$ is interior to $\Phi$. Actually, a cone denoted by $V(q)$, and its polar cone $N(q)$ in the sense of the standard scalar product of $\mathbb{R}^{n}$, will in the sequel be defined even for $q \notin \Phi$. When $q \in \Phi$, the cone $N(q)$ is nothing but the (outward) normal cone to $\Phi$ at this point (reduced to $\{0\}$ if $q$ is interior to $\Phi$ ).

$5^{\circ}$ The mechanical formulation of unilateral constraints has to encompass the geometric condition $\forall t \in l: q(t) \in \Phi$, together with some infomation about 
the associated forces of constraint. In the framework of traditional (smooth) Analytical Dynamics, this system of forces is represented, for every $t$, by its covariant components, say $r_{1}, \ldots, r_{n}$, relative to the generalized coordinates in use. This makes an element of $\mathbb{R}^{n}$ that we shall denote by $r$

The simplest case is that of frictionless contact. This classically means that the force of constraint at every possible point of contact is normal to the concerned bodies, with direction agreeing with unilaterality. One elementarily finds (see Sec.5 below) that, if all the considered unilateral constraints are of this sort, the whole information about them lets itself be summarized into the writing

$$
\forall t \in l: \quad q(t) \in \Phi \text { and }-r(t) \in N(q(t)) \text {. }
$$

(About the concept of a frictionless contact in the case of a less regular feasible region than above, see [10].)

Starting from (1.1), a decisive observation is made in Sec.5, namely that, for smooth motions, it implies the stronger assertion

$$
-r(t) \in \partial \psi_{v(q(t))}\left(u^{+}(t)\right)
$$

According to the usual notations of Convex Analysis, the right-hand member equals the normal cone at point $u^{+}(t)$ to the convex subset $V(q(t))$ of $\mathbb{R}^{n}$.

In addition, it is established that, if the initial data satisfy $q\left(t_{0}\right) \in \Phi$, then (1.2), assumed to hold for (dt-almost) every $t$, secures that $q(t)$ will remain in $\Phi$.

The advantage of (1.2) over (1.1) lies in the following. First, as we shall develop in Sec.5, this writing directly suggests some algorithms of timediscretization for computing the solutions to initial value problems. Secondly, by entering the velocity into the contact law, it paves the way to 
the consideration of friction. Furthermore, it is easily generalized to Nonsmooth Dynamics.

$6^{\circ}$ The function $t \rightarrow r(t) \in \mathbb{R}^{n}$ which, in the traditional Lagrange equations, represents the forces of constraints has, in Nonsmooth Dynamics, to be replaced by an $\mathbb{R}^{n}$-valued measure on the time-interval I, called the contact impulsion and denoted by $\mathrm{dR}$. For smooth motions, this measure admits the above function as its density relative to Lebesgue measure. A priori, there exists an infinity of representations of a vector measure, such as $O R$, in the form $d R=R_{\bigcup}^{\prime} d \mu$, where $d y$ is a nonnegative real measure and $R_{\hat{U}}^{\prime}$ a vectorvalued locally dy-integrable density function. We shall admit, as the law of frictionless contact in Nonsmooth Dynamics, the following generalization of (1.2), to be satisfied for every $t$ in 1 ,

$$
-R_{u}^{\prime}(t) \in \partial \psi_{Y(q(t))}\left(u^{+}(t)\right)
$$

Because the right-hand side is a cone, one shows that this condition is indifferent to the choice of the base measure dy. Furthermore, the existence of a function $R_{u}^{\prime}$ verifying (1.3) implies that $u^{+}(t)$ belongs to $v(q(t))$. Through Prop. 2.4 below, this ensures $q(t) \in \Phi$ for every $t$, provided the initial data satisfy $q\left(t_{0}\right) \in \Phi$, .

Assertion (1.3) about the contact impulsion makes the definition of the class of unilateral constraints that we call frictionless and soft. When transported into the equality of $\mathbb{R}^{n}$-valued measures, which governs Nonsmooth Dynamics, it generates a measure differential inclusion. The existence of solutions to the resulting initial value problems has so far been established only in some special cases [11][12][13] and is currently under investigation. The velocity jumps possibly occuring in such solutions are of the sort the author has previously called "standard inelastic shocks" [14] 
[15]. These are dissipative, so the corresponding evolution problems are essentially different from those one meets when the possible bounces are assumed "elastic" [16][17][18][19]. A synthetic view may be gained from the energy balance drawn in Sec. 10 below. The replacement of $u^{+}$in (1.2) by some weighted mean of $\mathrm{u}^{+}$and $\mathrm{u}^{-}$results in the introduction of a "dissipation index" $\delta$, with zero value in an elastic bounce, while the sof tness case corresponds to $\delta=1$.

$7^{\circ}$ Dry friction at a point of contact will be described by some extension of Coulomb's law to possibly anisotropic surfaces. The traditional formulation of this law rests on the decomposition of a contact force into its normal and tangential components; the formulation then consists of two separate statements respectively pertaining to zero and nonzero sliding velocity. In some of the author's early papers [20][21][22], it has been observed that, as soon as the normal component is treated as known, these pair of statements lets itself be synthetically expressed as a law of resistance deriving from a "pseudopotential". This in turn may be transcribed into a variational inequality [23], reflecting a "principle of maximal dissipation".

By a law of resistance, we mean a relation (in the present case, nonsmooth and not expressible through a single-valued function) between the contact force and the sliding velocity. Recall that significant mathematical and numerical papers have, in recent years, been devoted to problems which instead involve a "pseudo-friction" law. These problems are developed in the framework of small deviations and the sliding velocity vector is replaced by the tangential relative displacement of the contacting bodies. The status of such a pseudo-friction, compared to proper friction, is similar to that of Hencky plasticity with respect to proper plastic flow rules. 
The present lectures rest on a newer formulation of the possibly anisotropic Coulomb law, avoiding the decomposition of the contact force [24][25]. Similarly to what has been shown for the frictionless case, these formulations suggest numerical algorithms of time discretization. Furthermore, the resulting relation being conic with regard to the contact force, it admits an extension to Nonsmooth Dynamics, independent, as before, of the choice of a base measure du.

$8^{\circ}$ Here again, the possible nonsmooth motions are found to be governed by some measure differential inclusions. These differential inclusions are applied in Sec. 15 to the dynamics of velocity jumps.

Singularities in the dynamics of systems involving Coulomb friction used to be a matter of controversy during the first quarter of this century. It was observed that some initial value problems could admit several solutions or no solution and also that the behavlour of the investigated system depended on its constants on a discontinuous way. At the time, these findings were considered by such authors as P.Painlevé as contradicting the very bases of Physics. In modern views, nothing looks paradoxical in that, so there only remains of all these discussions the assertion, first made by L.Lecornu [26], that, in the presence of dry friction, velocity jumps are not necessarily the consequences of collisions.

It is shown by an example that the numerical techniques we propose can handle these frictional catastrophes without difficulty.

$9^{\circ}$ These lectures are restricted, for brevity, to time-independent constraints. However, by changing the reference frame and introducing adequate fictitious forces, one is able to apply the proposed methods to the motion of 
a small object lying on a vibrating table or, in the course of an earthquake, on the ground surface. An example is displayed, exhibiting some unexpected features.

$10^{\circ}$ For better agreement with the behaviour of real systems, one is commonly led to apply the traditional law of Coulomb with different values of the friction coefficient, depending on whether the sliding velocity vanishes or not. This distinction made between the "static" an "dynamic" friction coefficients seems, at first glance, to destroy the unity brought into the formulations by the use of Convex Analysis. Actually, it is shown in Sec. 17 that, far from beeing a mere empirical alteration of these formulations, such a distinction is inherently involved in the consequent developments. The numerical techniques proposed in these lectures are able to handle it without causing any computing problem. In fact, whether the sliding velocity exactly vanishes or not at the end of a time-step is explicitely determined by the algorithms; so the friction coefficient for the next step may be adjusted accordingly.

Let us close this Introduction by aknowledging that coulomb's law can provide only a rather crude approximation of the reality of dry friction (a recent review of the subject may be found in [27]). Also, the collisions affecting parts in real machines cannot be expected to fall exactly under one of the categories respectively described as "soft" or "elastic". And it is unlikely that any definite value of the "dissipation index" could be identified on a clear basis. But a fact of life is that, in most engineering situations, the higher order information needed for more accurate description is not available. So one has to be content with some moderately precise 
calculation, accounting at least for the main features of phenomena. In three years of experiments, the approach we propose has proved to be very workable. Because of their theoretical consistency and numerical stability, the described algorithms seem to be "robust" enough for accepting in the future various empirical alterations, aimed at improving their power of prediction.

No allusion is made in these lectures to the contact between deformable bodies, currently a very active domain of research. The reader will find references to this subject in other parts of this volume. In what concerns computation, since the spatial discretization of a continuous medium, using for instance a finite element scheme, generates a finite-dimensional space of positions, the design of numerical procedures may take an inspiration from the methods presented here (see e.g. [28]). But some fundamental differences between continuum dynamics and finite freedom dynamics have to be kept in mind. Because, in continuous media, every contact particle has zero mass, the concept of a soft contact, as opposed to an elastic contact, becomes unsignificant (it only stays as an option in constructing numerical algorithms). Possible dissipation reenters the scene through the constitutive laws which govern the behaviour of the concerned bodies. In elastic bodies, shock waves are expected to originate from boundary impacts. It is only when the time taken by these waves to travel the whole system is short, with respect to some other typical time values, that the treatment of deformable systems may be strictly conducted in the lines of finite freedom dynamics. Actually, most papers on continuous systems so far are restricted to quasistatic evolution problems, i.e. the terms involving inertia are neglected. 


\section{DIFFERENTIAL PROPERTIES}

Let a mechanical system have a finite number $n$ of degrees of freedom; every possible position of it may be located through the value it imparts to $q=\left(q^{1}, \ldots, q^{n}\right)$, an element of some open subset $\Omega$ of $\mathbb{R}^{n}$. This holds at least locally; in other words, $q^{1}, \ldots, q^{n}$ are local coordinates in the manifold of the system possible positions.

One defines a motion by making a depend on time. If the derivatives $q^{\prime}$ of the $n$ functions $t \rightarrow q^{i}$ exist at an instant $\tau$, we shall refer to the element $q^{\prime}=\left(q^{1}, \ldots, q^{n}\right)$ of $\mathbb{R}^{n}$ as the velocity of the system at this instant.

Motions will be studied on some time interval 1 , containing its origin $t_{0}$ but nonnecessarily closed nor bounded from the right. We shall not suppose the function $q: l \rightarrow \mathbb{R}^{n}$ derivable everywhere. Instead, we assume the existence of a velocity function $u: l \rightarrow \mathbb{R}^{n}$ such that

$$
\forall t \in l: \quad q(t)=q\left(t_{0}\right)+\int_{t_{0}}^{t} u(\tau) d \tau .
$$

This makes sense as soon as $u$ is locally Lebesgue-integrable on I. More speclally, we shall suppose that the function $u$ has locally bounded variation on I, l.e. It has bounded variation on every compact subinterval of I; notation: $u \in \operatorname{lbv}\left(1, \mathbb{R}^{n}\right)$. This secures that, at every $\tau$ in the interior of 1 , the right-limit $\mathrm{u}^{+}(\tau)$ and the left-limit $\mathrm{u}^{-}(\tau)$ exist.

By convention, for the initial instant $t_{0}$, the left-limit $u^{-}\left(t_{0}\right)$ is understood as equal to $u\left(t_{0}\right)$. This is more than a notational trick; such a writing actually reflects the significance we generally mean to give to the initial condition $u\left(t_{0}\right)=u_{0}$ of an evolution problem. It is intimated that investigation begins at $t_{0}$, but that the mechanical system was already in 
existence before. By $u_{0}$ is imparted some abridged information about the system history, precisely the left-limit $\mathrm{u}^{-}\left(\mathrm{t}_{0}\right)$.

Symmetrically, if I possesses a right end, say $t_{r}$, and contains it, the writing $u^{+}\left(t_{r}\right)=u\left(t_{r}\right)$ will prove convenient.

From (2.1) it results that the function a possesses at every $\tau>t_{0} a$ left-derivative $q^{-}(\tau)$, equal to $U^{-}(\tau)$ and, at every $\tau$ different from the possible right end of 1 , a right-derivative $q^{\prime}{ }^{+}(\tau)$, equal to $u^{+}(\tau)$.

In addition to the constraints which have permitted the q parametrization, we assume that the system is submitted to some unilateral constraints whose geometric effect is expressed by a finite set of inequalities

$$
f_{\alpha}(q) \leqslant 0, \quad \alpha \in[1,2, \ldots, \gamma] .
$$

The functions $f_{\alpha}: \Omega \rightarrow \mathbb{R}$ are supposed $C^{\prime}$ with respective gradients $\nabla f_{\alpha}=$ $\left(\partial f_{\alpha} / \partial q^{1}, \ldots, \partial f_{\alpha} / \partial q^{n}\right)$ different from zero, at least in a nelghbourhood of the corresponding hypersurface $f_{\alpha}=0$.

Inequalities (2.2) define the feasible region $\Phi$ of $\Omega$; for brevity, we assume that the functions $\mathrm{f}_{\alpha}$ do not depend on time, thereby leaving aside the possibility of moving constraints.

Through the chain rule, the existence of one-side derivatives for the functions $t \rightarrow q^{i}(t)$ implies the same for $t \rightarrow f_{\alpha}(q(t))$. Consequently, if a motion verifies $f_{\alpha}(q(t)) \leqslant 0$ for every $t$, then at any instant $\tau$ such that $f_{\alpha}(q(\tau))=0$, one readily finds $u^{+}(\tau) . \nabla f_{\alpha}(q(\tau)) \leqslant 0$ and $u^{-}(\tau) \cdot \nabla f_{\alpha}(q(\tau)) \geqslant 0$ (the dot refers to the usual scalar product of $\mathbb{R}^{n}$ ). 
Generally, let us put:

NOTATION 2.1 For every $q \in \Omega$ define

$$
J(q):=\left\{\alpha \in\{1, \ldots, \gamma\}: f_{\alpha}(q) \geqslant 0\right\}
$$

and

$$
V(q):=\left\{v \in \mathbb{R}^{n}: \forall \alpha \in J(q), v \cdot \nabla f_{\alpha}(q) \leqslant 0\right\}
$$

robserve that $\mathrm{V}(\mathrm{q})$ equals the whole of $\mathbb{R}^{n}$ if $\mathrm{J}(\mathrm{q})=\varnothing$ ).

Using as above the one-side derivatives, one obtains:

PROPOSITION 2.2 If a motion $\mathrm{t} \rightarrow \mathrm{q}(\mathrm{t})$ agrees with the set of constraint inequalities (2.3), i.e. $\mathrm{q}(\mathrm{t}) \in \Phi$ for every $\mathrm{t}$, then

$\forall t \in \operatorname{int} 1: \quad u^{+}(t) \in V(q(t))$ and $u^{-}(t) \in-V(q(t))$.

REMARK 2.3 In existential studies as well as in numerical algorithms, the definition (2.4) of $V(q)$ will commonly be invoked with $q \notin \Phi$. Then, the following is useful:

PROPOSITION 2.4 Let the function $t \rightarrow q(\mathrm{t})$ be associated with some $u \in \mathcal{L}^{\prime}{ }_{\operatorname{loc}}\left(1, \mathbb{R}^{n}\right)$ through (2.1). Suppose that $\mathrm{q}\left(\mathrm{t}_{\mathrm{o}}\right) \in \Phi$ and that, for Lebesguealmost every $\mathrm{t} \in \mathrm{l}$, one has $\mathrm{u}(\mathrm{t}) \in \mathrm{V}(\mathrm{q}(\mathrm{t}))$. Then $\mathrm{q}(\mathrm{t}) \in \Phi$ for every $\mathrm{t}$.

PROOF. Let us suppose the existence of some $\tau \in I$, with $q(\tau) \notin \Phi$ and look for contradiction. There exists $\alpha \in\{1, \ldots, \gamma\}$ such that $f_{\alpha}(q(\tau))>0$. The set $\{t \in l: t \leqslant \tau$ and $\left.f_{\alpha}(q(\tau)) \leqslant 0\right\}$ is nonempty (it contains $t_{0}$ ); let $\sigma$ denote its l.u.b. Due to the continuity of $f_{\alpha}$, one has $f_{\alpha}(q(\sigma))=0$. Since $f_{\alpha}$ is $C^{\prime}$, the function $t \rightarrow f_{\alpha}(q(t))$ is absolutely continuous on $[\sigma, \tau]$; after expressing its derivative by the chain rule, one may write

$$
f_{\alpha}(q(\tau))=\int_{\sigma}^{\tau} u(t) \cdot \nabla f_{\alpha}(q(t)) d t
$$


In view of the definition (2.4) of $V$, the integrand should be $\leqslant 0$ for Lebesgue-almost every $t$, hence $f_{\alpha}(q(\tau)) \leqslant 0$, which is a contradiction.

If $u$ has locally bounded variation, it belongs to $\mathcal{L}^{\prime}{ }_{100}\left(1, \mathbb{R}^{n}\right)$ and the set of its discontinuity points is countable, hence Lebesgue-negligible. Thus, in using the above Proposition, one may replace $u$ in the assumption $u(t) \in V(q(t))$ by $u^{+}$or $u^{-}$or any weighted mean of them.

REMARK 2.5 The subset $V(q)$ of $\mathbb{R}^{n}$ is a closed convex cone. In case $q \in \Phi$, this coincides with what is usually called the tangent cone to the region $\Phi$ at point $q$ (equal, in particular, to the whole of $\mathbb{R}^{n}$ if $q \in$ int $\Phi$ ). On the contrary, if $q \notin \Phi$, one commonly agrees to say that the tangent cone to $\Phi$ at this point is empty; so is not $\mathrm{V}(\mathrm{q})$.

Some caution is needed when interpreting the concept of a tangent cone. Let $a_{0} \in \Phi$ and $v \in \mathbb{R}^{n}$; in view of Prop.2.2, for the existence of a mapping $q: 1 \rightarrow \Phi$ such that $q\left(t_{0}\right)=a_{0}$ and $q^{\prime+}\left(t_{0}\right)=v$, it is necessary that $v \in V\left(a_{0}\right)$; $a$ counter-example may be found in [15], showing that this is not sufficient. However, if in addition one assumes int $V\left(q_{0}\right) \neq \varnothing$, then existence is secured [29]. Through classical Convex Analysis, the latter assumption is equivalent to the polar cone of $\mathrm{V}\left(\mathrm{q}_{0}\right)$ having a compact basis; this is the convex cone generated in $\mathbb{R}^{n}$ by the elements $\nabla f_{\alpha}\left(q_{0}\right)$, with $\alpha \in J\left(q_{0}\right)$, so the assumption amounts to assert the existence of a hyperplane in $\mathbb{R}^{n}$, not containing the origin, which intersects all the half-lines generated by these elements. We shall meet this cone again in Sec.5.

REMARK 2.6 A deeper insight into the situation could be gained by 
considering the differential manifold $\mathcal{P}$ of the system positions, without preference to any peculiar system of local coordinates. A motion is the conceived as a mapping $p: 1 \rightarrow \mathcal{P}$. The (possibly one-side) velocity of the system at some instant $\tau$ equals, by definition, the (possibly one-side) derivative of this mapping, an element, say $p^{\prime}(\tau)$, of the tangent space $\mathcal{P}_{p(\tau)}^{\prime}$ to $\mathcal{P}$ at point $p(\tau)$. The real numbers $q^{i}(\tau)$ considered in the foregoing equal the components of $p^{\prime}(\tau)$, relative to the base induced in $\mathcal{P}_{p(\tau)}^{\prime}$ by the local coordinate system in use. Inequalities (2.2) are imparted a coordinate-free meaning provided one understands the functions $f_{\alpha}$ as $C^{\prime}$ mappings of $\mathcal{P}$ to $\mathbb{R}$, without reference to any choice of local coordinates. Then, by the gradient $\nabla f_{\alpha}(p)$ is meant an element of $\mathcal{P}_{p}^{* *}$, the cotangent space to $\mathcal{P}$ at point $p$. Also in this linear space, the local coordinate system induces a base; the partial derivatives precedingly invoked equal the components of $\nabla f_{\alpha}(p)$ relative to this base. In Definition (2.4), the Euclidean scalar product of $\mathbb{R}^{n}$ should then be replaced by the bilinear form $\langle\ldots$,$\rangle which puts the linear$ spaces $\mathcal{P}_{p}^{\prime}$ and $\mathcal{P}_{p}^{* *}$ in duality. Thereby, for every position $p$, a pair of mutually polar convex cones is defined in these dual linear spaces, without reference to any cholce of local coordinates.

The concept of a convex cone in $\mathcal{P}_{p}^{\prime}$ or in $\mathcal{P}_{p}^{\prime *}$ is meanigful, in view of the linear structure of these tangent spaces. Concerning, on the contrary, the feasible region, the differentiable manifold $\mathcal{P}$ cannot in general support any convexity assumption. If such an assumption is made, it only refers to some peculiar coordinate system. However, let us mention the following special case.

For the treatment of dynamical problems concerning a scleronomic 
system, the expression $\frac{1}{2} A_{i j}(q) q^{\prime} q^{\prime j}$ of the kinetic energy has to be introduced. This is a positive definite quadratic form in $q^{\prime}$ and, classically, by putting $d s^{2}=A_{1 j}(q) d q^{i} d q^{j}$, one equips the differential manifold $\mathcal{P}$ with a Riemannian metric independent of the coordinates in use. So this metric is intrinsically associated with the dynamical structure of the mechanical system. Now, it may happen that some local coordinates exist, such that the coefficients $A_{i j}(q)$ are constant in $q$; so is the case, for instance, if the system consists of a single rigid body performing only motions parallel to a fixed plane. Under such circumstances, the curvature of $\boldsymbol{P}$ is zero; in other words, this manifold is locally Euclidean. Then, at least in sufficiently small regions, the concept of the convexity of a subset of $\mathcal{P}$ becomes mechanically meaningful. The mathematical paper [16] was precisely based on the convexity of the feasible region.

Anyway, the writing in (2.1) makes sense only as far as the functions $t \rightarrow q$ and $t \rightarrow u$ take their values in a fixed linear space, namely $\mathbb{R}^{n}$ for the present. On the contrary, in the differential geometric setting, the velocity at time $t$ would be an element of the tangent space $\mathcal{P}_{p(t)}^{\prime}$, which depends on $t$ through the unknown mapping $\mathrm{p}: \mathrm{l} \rightarrow \boldsymbol{P}$.

\section{KINEMATICS}

In all the sequel, each of the inequalities $f_{\alpha} \leqslant 0$ will be understood as characterizing the system positions agreeing with the mutual impenetrability of a certain pair of rigid bodies. For instance, let us drop the subscript $\alpha$ and assume that condition $f \leqslant 0$ expresses that some rigid part $B_{1}$ of the system does not overlap a given external obstacle $\mathcal{B}_{0}$, fixed relative to the 
reference frame in use. The impenetrability of two rigid bodies $\mathcal{B}_{1}$ and $\mathcal{B}_{2}$, which both are constituents of the system, would finally result in the same formalism (see [30], where the case of an external obstacle with prescribed motion is also considered).

Equality $f(q)=0$ means that, in the position $q$ of the system, the part $\mathcal{B}_{1}$ touches $\mathbb{B}_{0}$. We shall always assume that contact takes place through a single particle of $\mathcal{B}_{1}$, which in general depends on $q$, say $\mathcal{M}_{1}(q)$. The respective boundaries of contacting bodies will be supposed to permit the definition of a common tangent plane at $\mathcal{M}_{1}(q)$ to these boundaries. This does not preclude edges or vertices; one of the bodies may even reduce to a single particle, provided the boundary of the other is a smooth surface.

Let $\mathcal{N}_{\mathrm{q}}$ denote the unit vector, normal to this tangent plane and directed toward $\mathcal{B}_{1}$.

As usual, the primitive constraints of the system, i.e. the constraints which have permitted the parametrization through $\left(q^{1}, \ldots, q^{n}\right)$, are assumed smooth enough for the following to hold. Let a motion be described by giving $q$ as a function of $t$. For every $t$ such that the (possibly one-side) derivatives $q^{1}{ }^{1}, \ldots, q^{n}$ exist, every particle, say $\mathcal{M}$, of the system possesses a (possibly one-side) velocity vector, relative to the reference frame in use. Calculating this vector yields an expression $\mathcal{V}\left(\mathcal{M}, q, q^{\prime}\right)$, aff ine with regard to $q^{\prime}$.

For brevity, we shall restrict the sequel to the scleronomic case, l.e. the primitive constraints do not depend on time; then the above expression is linear in q'. Let us apply this to the contact particle $\mathcal{M}_{1}(q)$ and put the notation

$$
G_{q} q^{\prime}:=V\left(\mathcal{M}_{1}(q), q, q^{\prime}\right)
$$


For every q such that $f(q)=0$, this introduces the mapping $q^{\prime} \rightarrow \mathcal{G}_{q} q^{\prime}$, linear of $\mathbb{R}^{n}$ to the Euclidean linear space $\mathcal{F}_{3}$ of the vectors of physical space.

In the case of a pair of bodies which both are parts of the system, a linear mapping similar to $G_{\mathrm{q}}$ would express from $q^{\prime}$ the relative velocity, at a possible contact point, of one of this part with respect to the other.

The writing in (3.1) is not restricted to motions agreeing with the impenetrability constraint. We now are to take this constraint into account. Let a value of $q$ correspond to contact, i.e. $f(q)=0$, and let $v \in \mathbb{R}^{n}$. Let a motion start from this position $q$ at some time $\tau$, with right-velocity $q^{\prime+}$ equal to $v$ at this instant. Evaluating $f$ at all subsequent positions, one obtains a function of time whose right-derivative at $\tau$ may be expressed through the chain rule, namely v. $\nabla f(q)$. Assume $v . \nabla f(q)<0$; then instant $\tau$ is followed by a nonzero time interval over which $f<0$, i.e. $\mathcal{B}_{1}$ and $\mathcal{B}_{0}$ break contact. This implies that, at $\tau$, the right-velocity $G_{q} \vee$ of the contact particle $\mathcal{M}_{1}(q)$ of $\mathcal{B}_{\text {, verifie }} \mathcal{N}_{\mathrm{q}} \cdot \mathcal{G}_{\mathrm{q}} \vee \geqslant 0$; otherwise the motion of this particle would require of $\mathcal{B}_{1}$ to overlap $\mathcal{B}_{0}$, so making $f \geqslant 0$.

This shows that, for $v \in \mathbb{R}^{n}$ and for any fixed $q$ satisfying $f(q)=0$, one has the implication

$$
v \cdot \nabla f(q)<0 \Rightarrow \mathcal{N}_{q} \cdot G_{q} \vee \geqslant 0 .
$$

Let us introduce now the linear mapping $G_{q}^{*}: \mathbb{E}_{3} \rightarrow \mathbb{R}^{n}$, the transpose of $G_{q}$ in the sense of the Euclidean autodualities of $\mathcal{E}_{3}$ and $\mathbb{R}^{n}$; then $\mathcal{N}_{q} \cdot \mathcal{G}_{q} \vee=$ v. $G_{q}^{*} \mathcal{N}_{q}$. Recall that we have assumed $\nabla f \neq 0$; through a unflateral version of the Lagrange multiplier theorem, implication (3.2) yields:

PROPOSITION 3.1 For every $q$ verifying $f(q)=0$, there exists $\lambda \geqslant 0$ such that 


$$
\mathcal{G}_{q}^{*} \mathcal{N}_{q}=-\lambda \nabla f(q)
$$

REMARK 3.2 We shall later need that the element $G_{q}^{*} \mathcal{N}_{q}$ of $\mathbb{R}^{n}$ differ from zero, i.e. $\lambda>0$. It is a general fact that the kernel of $G_{q}^{*}$ equals the subspace of $\mathcal{E}_{3}$ orthogonal to the range $G_{q}\left(\mathbb{R}^{n}\right)$ of $G_{q}$. In particular, when $G_{q}\left(\mathbb{R}^{n}\right)$ is the whole of $\mathcal{E}_{3}$, the kernel of $G_{q}^{*}$ reduces to $\{0\}$ and this secures $G_{q}^{*} \mathcal{N}_{q} \neq 0$. But, in some usual applications, $G_{q}\left(\mathbb{R}^{n}\right)$ will be a strict subspace of $\mathcal{E}_{3}$; for instance, if the primitive constraints allow $\mathcal{B}_{1}$ to perform only motions parallel to some fixed plane, then $\operatorname{dim} G_{q}\left(\mathbb{R}^{n}\right)=2$. What precedes shows that $\mathcal{G}_{q}^{*} \mathcal{N}_{q}=0$ if and only if $\mathcal{G}_{q}\left(\mathbb{R}^{n}\right)$ is contained in the two-dimensional subspace of $\mathcal{E}_{3}$ consisting of the vectors parallel to the common tangent plane to contacting bodies.

REMARK 3.3 So far, $G_{q}$ has been defined only for such $q$ that $f(q)=0$. In computation, as well as in existential studies, it will prove useful to extend the definitions of $\mathcal{G}_{\mathbf{q}}$ and $\mathcal{N}_{q}$, in a smooth arbitrary way, to the whole range $\Omega$ of the local coordinates in use, or at least to some neighbourhood of the hypersurface $f=0$. This extension may additionally be required to preserve the property (3.3).

REMARK 3.4 Let a motion comply with condition $f \leqslant 0$ at every time. Consider an instant of contact, l.e. at which $f(q)=0$, and suppose that the two-sided derivative $q$ exists at this instant. Since the latter equals the common value of $q^{+}$and $q^{\prime}$, the observations made in $\operatorname{Sec} .2$ imply $q^{\prime} . \nabla f(q)=0$. In view of Proposition 3.1 , this yields $\mathcal{N}_{\mathrm{q}} \cdot G_{\mathrm{q}} q^{\prime}=0$, expressing that the velocity of the particle $\mathcal{M}_{1}(q)$ of $\mathcal{B}_{1}$ through which this body touches the fixed obstacle $\mathbb{B}_{0}$ is a vector parallel to the common tangent plane. 
Similar reasoning would apply to a pair of moving parts of the system: if contact holds at some instant and if, at this instant, the time-derivative $q^{\prime}$ exists, in the ordinary two-side sense, then the relative velocity of one of these bodies with respect to the other, at the contact point, is a vector parallel to the common tangent plane. The same fact is classically established in elementary Kinematics, under the stronger assumption of permanent contact. One refers to this relative velocity as the sliding velocity of the first body upon the second.

\section{LAGRANGE EQUATIONS}

Let a motion of the system be described under the form (2.1). It will be said smooth if the velocity function $u$ is locally absolutely continuous, i.e. absolutely continuous on every compact subinterval of 1 . This implies the existence of the derivative $u^{\prime}(t)=q^{\prime \prime}(t)$ for Lebesgue-almost every $t$. We shall refer to the element $q^{\prime \prime}$ of $\mathbb{R}^{n}$ as the acceleration of the system.

Such a motion agrees with Dynamics if and only if the function $t \rightarrow q(t)$ verifies the Lagrange differential equation

$$
\frac{d}{d t}\left(\partial \mathcal{E}_{c} / \partial q^{\prime}\right)-\partial \mathcal{E}_{c} / \partial q^{i}=c_{i}
$$

Here $\mathcal{E}_{c}\left(q, q^{\prime}\right)$ denotes the expression of the kinetic energy; since we assume the system scleronomic, this is

$$
\mathcal{E}_{c}\left(q, q^{\prime}\right)=\frac{1}{2} A_{i j}(q) q^{\prime i} q^{\prime j},
$$

a positive definite quadratic form in $q^{\prime}$.

By $c_{i}$ are denoted the covariant components of the totality of the forces acting on the system. These possibly comprise some given forces, whose covariant components $F_{i}$ are known functions of time, position and 
velocity, and also comprise the a priori unknown reactions or constraint forces, involved in the constraints that the system experiences. As usual, when Lagrange equations are applied, we shall assume that the primitive constraints, l.e. those which have permitted the q parametrization, are perfect, in the sense that the corresponding reactions have zero covariant components. But we shall have to take into account the reactions of the superimposed unllateral constraints.

The left-hand side of (4.1) may be developed into

$$
A_{i j} q^{n j}+\left(A_{i j, k}-\frac{1}{2} A_{j k, i}\right) q^{j} q^{k} \text {, }
$$

where $A_{1 j, k}$ denotes the partial derivative of $A_{1 j}$ with regard to $q_{k}$. Therefore, (4.1) takes on the form $A_{i j}{ }^{a^{j}}=K_{i}+r_{i}$; here the term $r_{i}$ refers to the totality of the reactions of superimposed constraints, while $K_{i}$ is a known function of $t, q, q$, equal to $F_{i}$ minus all the terms in (4.3) which involve $q^{j} q^{k}$. Since the matrix $A$ is invertible, we may finally write this down as

$$
q^{\prime \prime}=A^{-1} K+A^{-1} r \text {. }
$$

It has been assumed that each inequality $f_{\alpha}(q)=0$ expresses the contact between some pair of rigid bodies. Let us denote by $r_{1}^{\alpha}, \ldots, r_{n}^{\alpha}$ the covariant components of the corresponding reaction, making an element of $\mathbb{R}^{n}$ denoted by $r^{\alpha}$. Formally, this term will be introduced also when the said contact is not in effect, so we shall state

$$
f_{\alpha}(q)<0 \Rightarrow r^{\alpha}=0 \text {. }
$$

To fix the ideas, suppose, as in Sec.3, that the contact expressed by $f_{\alpha}(q)=0$ takes place between some part, say $\mathcal{B}_{\alpha}$, of the system and an unmoving external obstacle. Suppose that the contact action on $\mathcal{B}_{\alpha}$ results in 
a single force $\mathbb{R}^{\alpha}$, applied to a particle of this body denoted by $\mathcal{M}_{\alpha}(q)$. By definition, the covariant components of this force make the element $r^{\alpha}$ of $\mathbb{R}^{n}$ such that

$$
\forall v \in \mathbb{R}^{n}, \mathcal{R}^{\alpha} \cdot \mathcal{V}\left(\mathcal{M}_{\alpha}(q), q, v\right)=r^{\alpha}, v .
$$

On the left-hand side, the dot refers to the Euclidean scalar product of $\mathbb{F}_{3}$, on the right-hand side to the standard scalar product of $\mathbb{R}^{n}$. As before, due to scleronomy, $\mathcal{V}\left(\mathcal{M}_{\alpha}(q), q, v\right)$ is a linear expression in $v$, say $\mathcal{G}_{q}^{\alpha} v$. Then, by introducing the transpose mapping $G_{q}^{\alpha *}: \mathbb{E}_{3} \rightarrow \mathbb{R}^{n}$, one equivalently writes down (4.6) as

$$
r^{\alpha}=G_{q}^{\alpha *} R^{\alpha}
$$

\section{SMOOTHFRICTIONLESS MOTIONS}

In this Section, we shall assume that the possible unilateral contacts are frictionless. Under the preceding notations, this means that, for every $\alpha$ such that $f_{\alpha}(q)=0$,

$$
\exists \rho \in \mathbb{R}: \mathcal{R}^{\alpha}=\rho \mathcal{N}_{a}^{\alpha},
$$

where $\mathcal{N}_{q}^{\alpha}$ denotes the common normal unit at $\mathcal{M}_{\alpha}(q)$ to the contacting bodies, directed toward $\mathcal{B}_{\alpha}$.

We shall assume in addition that the contact is unilateral without adhesive effect, i.e. $0 \geqslant 0$

Through Prop.3.1, conditions (4.7) and (5.1) imply

$$
\exists \mu_{\alpha} \geqslant 0: r^{\alpha}=-\mu_{\alpha} \nabla f_{\alpha}(q) \text {. }
$$

Note that the repetition of a Greek index will never be understood as implying summation.

Provided that $G_{q}^{\alpha *} \mathcal{N}_{q}^{\alpha} \neq 0$ (see Remark 3.2), it may more precisely be 
checked that (5.2) is equivalent to the existence of $R^{\alpha}$ agreeing with the above assumptions. And, in view of the convention made in (4.5), this equivalence remains valid for every $q \in \Phi$ if one stipulates that $\mu_{\alpha}=0$ when $f_{\alpha}(q)<0$. Furthermore, in formulating our evolution problem under the geometric condition $a \in \Phi$, it is immaterial to state that (5.2) also holds for non feasible $q$.

Therefore, a value of the total reaction term $r=\sum r^{\alpha}$ is compatible with the stated laws of contact if and only if it satisfies

$$
r \in-N(q) \text {, }
$$

where $N(q)$ denotes the convex cone generated in $\mathbb{R}^{n}$ by the elements $\nabla f_{\alpha}(q)$, $\alpha \in J(q)$ (see Notation 2.1). According to an usual convention, if $J(q)=\varnothing$ this cone consists of the zero of $\mathbb{R}^{n}$. In all cases $V(q)$, as defined in (2.4), and $N(q)$ make a pair of mutually polar or conjugate cones. When a belongs to the feasible region, N(q) is nothing else than the (outward) normal cone to $\Phi$ at point $q$, but what we are denoting here by $N(q)$ also makes sense and is nonempty for $q \notin \Phi$.

Eliminating $r$ between (5.3) and the Lagrange equations, as they are displayed in Sec.4, one obtains that a smooth motion of the system agrees with all the mechanical conditions stated, if and only if the differential inclusion

$$
-A(q) q^{\prime \prime}+K\left(t, q, q^{\prime}\right) \in N(q)
$$

is satisfied Lebesgue-a.e. in I, together with the geometric condition of the unilateral constraints,

$$
\forall t \in l: \quad q(t) \in \Phi .
$$

The Proposition below marks a turning point, regarding all our subject 
matter. For every (closed, convex) subset of $\mathbb{R}^{n}$, say $C$, we denote by $\Psi_{C}$ its indicator function, i.e. $\Psi_{C}(x)=0$ if $x \in C$ and $+\infty$ otherwise. The subdifferential $\partial \Psi_{C}(x)$ is known to equal the normal cone to $C$ at point $x$ (empty if $x \notin C$ ).

PROPOSITION 5.1 A smooth motion, with initial data $\mathrm{q}\left(\mathrm{t}_{0}\right)$ belonging to $\Phi$, is a solution or (5.4), (5.5) if and only if the velocity function $u$ associated with a through (2.1) satisfies Lebesgue-a.e. in I the differential inclusion

$$
-A(q) u^{\prime}+K(t, q, u) \in \partial \psi_{v(q)}(u)
$$

PROOF. For every $t$ such that (5.6) holds, the right-hand side is nonempty, hence $u(t) \in V(q(t))$. Since, by assumption, $u$ is a (locally absolutely) continuous function, (2.1) entails that $u(t)$ equals, for every teint $I$, the (twosided) derivative of $t \rightarrow q(t)$ Through Proposition 2.4, one concludes that, if (5.6) is verified Lebesgue-a.e., then (5.5) holds. Furthermore, (5.6) implies (5.4) because $\partial \psi_{V(q)}(u)$ is essentially a subset of $N(q)$, the polar cone of $V(q)$.

Conversely, suppose that the function $t \rightarrow q(t)$ satisfies (5.5). Since $u$ is continuous, Proposition 2.2 shows that $u(t)$, for every $t \in i n t I$, belongs to $V(q(t)) \cap-V(q(t))$ which is the linear subspace of $\mathbb{R}^{n}$ orthogonal to $N(q(t))$. Therefore, if (5.4) holds Lebesgue-a.e., then for Lebesgue-almost every $t$, the values of $u$ and $-A U^{\prime}+K$ are orthogonal and respectively belong to the pair of mutually polar cones $\mathrm{V}(\mathrm{q}(\mathrm{t}))$ and $\mathrm{N}(\mathrm{q}(\mathrm{t}))$; consequently they are conjugate points relative to this pair, i.e. (5.6) holds.

REMARK 5.2 At the present stage, where the motion smoothness, i.e. the local absolute continuity of $u$, is a priori assumed, the same symmetry between past and future is observed as in the classical case of bilaterally 
constrained frictionless systems. In particular, for the differential inclusion (5.6) to take care automatically of condition $q \in \Phi$, it is enough that $q(\tau)$ belongs to $\Phi$ at some $\tau \in I$, nonnecessarily the initial instant. Also an equivalence similar to what is stated in the above Proposition may symmetrically be established, with (5.6) replaced by

$$
-A(q) u^{\prime}+K(t, q, u) \in-\partial \psi_{-}-v(q)(u) \text {. }
$$

Similarly to (5.6), this implies the orthogonality of the elements $u$ and $-A u^{\prime}+K$ of $\mathbb{R}^{n}$. From such an orthogonality, the same power equation may be derived as in the traditional case of frictionless time-independent bilateral constraints:

$$
\frac{\mathrm{d}}{\mathrm{dt}} E_{c}=F \cdot u
$$

REMARK 5.3 As an introduction to forthcoming Sections, let us indicate how the formulation (5.6) directly suggests a procedure of time discretization for computing approximately the motion consequent to some initial data

$$
\begin{array}{ll}
q\left(t_{0}\right)=q_{0}, & \text { given in } \Phi \\
u\left(t_{0}\right)=u_{0}, & \text { given in } v\left(q_{0}\right) .
\end{array}
$$

Let $\left(t_{1}, t_{F}\right)$ be a time-step (here 1 is understood as referring to "initial" and $F$ to "final"), with length $n=t_{F}-t_{1}$ and midpoint $t_{M}=t_{1}+\frac{1}{2} n$. From the approximate values $q_{1}, u_{1}$ of the functions $q$ and $u$ at $t_{1}$, one has to compute $q_{F}$ and $u_{F}$, corresponding to $t_{F}$.

Using $\left(u_{F}-u_{l}\right) / h$ as an approximant of $u^{\prime}$, one discretizes the differential inclusion (5.6) into

$$
-A\left(q_{M}\right)\left(u_{F}-u_{1}\right) / h+K\left(t_{M}, q_{M}, u_{1}\right) \in \partial \psi_{M}\left(u_{F}\right)
$$

Here $q_{M}=q_{1}+\frac{1}{2} h u_{1}$ is a midpoint approximant of $q_{\text {; }}$ by $\psi_{M}$ is denoted the 
indicator function of $V\left(q_{M}\right)$. Inserting $u_{F}$ as an approximant of $u$ in the right-hand side tends to qualify this discretization scheme as "implicit". However, the smoothness of the given function Kallows one to replace in it $u$ by $u_{1}$, so the procedure may be said "semi-implicit".

We shall come back later to more general algorithms of the same sort; let us only show here how (5.11) uniquely determines $u_{F}$. Suppose, for simplicity, that $A\left(q_{M}\right)$ reduces to the unit matrix. This actually entails no loss of generality: in the line of Remark 2.6, it amounts to make of the tangent space, at the point $q_{M}$ of the position manifold, a Euclidean linear space, with scalar product defined through $A\left(q_{M}\right)$, and to take an orthonormal base in this space (more detail on the practical use of this trick may be found in $(15])$. Then (5.11) becomes

$$
-\left(u_{F}-u_{1}\right)+h K \in \partial \Psi_{M}\left(u_{F}\right)
$$

The multiplication of both members of (5.11) by the positive number $h$ has not altered the right-hand side, because $\partial \psi_{M}\left(u_{F}\right)$ is a cone. In view of the classical characterization of the proximal point to $u_{1}+h K$ in the closed convex subset $V\left(q_{M}\right)$ of the Euclidean linear space, this is equivalent to

$$
u_{F}=\operatorname{prox}\left(u_{1}+n K, v\left(q_{M}\right)\right) \text {. }
$$

observe that $u_{1}+h K$ is nothing but the value that $u_{F}$ would take in the case $J\left(a_{M}\right)=\varnothing$, i.e. the value that the discretization of Lagrange equations would yield in the absence of superimposed unilateral constraints.

After determining $u_{F}$, one finishes the computation step by calculating $a_{F}=a_{M}+\frac{1}{2} h u_{F}$. 


\section{PERCUSSIONS AND FRICTIONLESS SHOCKS}

The preceding Section was restricted to motions a priori assumed smooth. For such a motion, there may exist in particular a time interval during which one or more of the contacts persist, say the contact expressed by $f_{\alpha}=0$. Call $t_{c}$ the end of this interval and suppose that a nonzero interval follows, over which $f_{\alpha}<0$. In other words, as soon as $t$ exceeds $t_{c}$, the set $J(q(t))$ ceases to contain $\alpha$; thus the cone $V(q(t))$ suddenly increases. This involves no contradiction with the assumed (locally absolute) continuity of $u$, nor with the inclusion uevn-V, resulting from Prop.2.2. Certain motions of a unilateral pendulum provide familiar examples of this.

Computationally, there is no difficulty in approximating a motion showing such a smooth contact break, through the algorithm of Remark 5.3. It only happens that, from a certain time-step to the next, the dimension of $V\left(a_{M}\right)$ suddenly increases, without producing any notable irregularity in the sequence of the calculated values of $u$.

Imagine, on the contrary, that an interval of smooth motion ends at some instant $t_{s}$ with the occurence of new contacts, f.e. $J(q)$ suddenly increases. In view of Prop.2.2, the continuity of $u$ at $t_{s}$ would require of the left-limit $u^{-}\left(t_{s}\right)$, an element of $-V\left(q\left(t_{s}\right)\right)$, to belong also to $V\left(\alpha\left(t_{s}\right)\right)$. This would mean that the new contacts are attained tangentially, an event which cannot be expected in general. So, a discontinuity of $u$ at $t_{s}$ has to be contemplated. This is called a shock and, to deal with it, Classical Mechanics provides the concept of percussion, that we are to review in a few words.

Assume that $t_{s}$ is followed by another interval of smooth motion. It will be understood that, because of slight deformability in the system parts, the 
velocity change is not rigorously instantaneous, but takes place on a "very short" time interval, say $\left(t_{s}, t_{s}+\theta\right)$, over which the differential equations of smooth dynamics supposedly hold. In view of the steep velocity change, these equations are expected to involve "very large" values of the contact forces. By equalling the integrals on $\left(t_{s}, t_{s}+\theta\right)$ of both members of the differential equations, one obtains the momentum change formula. This is a balance equation, through which the net velocity change is related to the time integral, say $\Pi$, of the function $t \rightarrow r(t) \in \mathbb{R}^{n}$ which, in the notations of Sec.4, represents the contact forces. Compared to it, the term $K$ yields a negligible integral, because $\theta$ is "very small"; for the same reason, the variations of q on the interval are neglected. Once obtained, the balance formula is inserted into the former setting of instantaneous shock; the element $\pi$ of $\mathbb{R}^{n}$ is then said to make the components of the contact percussions. This procedure may be seen as an early example of a multiple scaling; it permits to calculate the after-shock velocity $u^{+}\left(t_{s}\right)$, as far as sufficient information is avallable about contact percussions.

Usually, the above reasoning is applied under the assumption of frictionless contacts. Then it seems natural to admit, similarly to (5.2), that, for every $\alpha \in J\left(q_{S}\right)$, the contribution to $\pi$ of the corresponding contact, say $\pi^{\alpha}$, has the following form

$$
\exists M_{\alpha} \geqslant 0: \Pi^{\alpha}=-M_{\alpha} \nabla f_{\alpha}\left(q\left(t_{s}\right)\right) .
$$

The argument commonly proposed in support to this assertion about $\pi^{\alpha}$ is that, in (5.2), the vector $\nabla f_{\alpha}(q(t))$ should remain nearly constant during the very short time interval $\left(t_{s}, t_{s}+\theta\right)$, because the variations of $q$ are very small; thus $M_{\alpha}$ would simply equal the integral of the nonnegative real function 
$t \rightarrow \bigcup_{\alpha}(t)$. In the author's opinion, this conclusion cannot be accepted without further discussion, though physical situations certainly exist in which (6.1) accurately agrees with reality. In fact, the use of the equations of regular Dynamics rests on the smoothing effect of a certain amount of deformation in the system parts. The very meaning of the parametrization q may then be questioned. Furthermore, the resulting alterations of the functions $f_{\alpha}$, however small in amplitude and possibly concentrated in the vicinity of the point $\mathrm{q}\left(\mathrm{t}_{\mathrm{s}}\right)$, are prone to generate nonnegligible variations of the vectors $\nabla f_{\alpha}(q(t))$ in the course of the interval $\left(t_{s}, t_{s}+\theta\right)$. A similar discussion could also be conducted on equations (4.7) and (5.1): the deformation of the contacting bodies, however small, may appreciably alter the vector $\mathcal{N}_{q}^{\alpha}$ in $\mathfrak{E}_{3}$ as well as the mapping $G_{q}^{\alpha *}: \mathcal{E}_{3} \rightarrow \mathbb{R}^{n}$.

At all events, (6.1) does not bring enough information about $\pi$ to determine $\mathrm{u}^{+}\left(\mathrm{t}_{\mathrm{s}}\right)$ completely, even in the simplest case where $J\left(q\left(t_{s}\right)\right)$ consists of a single element. Classically, a shock (in a scleronomic system) is said elastic if it preserves the total kinetic energy; this additional assertion permits, in the case of a single contact, to determine $u^{+}\left(t_{3}\right)$ unambiguously. This may be given a geometric form by using in $\mathbb{R}^{n}$ the kinetic metric, i.e. the Euclidean metric defined by the matrix $A\left(q\left(t_{s}\right)\right)$. Then, if $J\left(q\left(t_{s}\right)\right)=\{\alpha\}$, one finds that $u^{+}\left(t_{s}\right)$ equals the mirror image of $u^{-}\left(t_{s}\right)$ relative to the tangent plane at the hypersurface $f_{\alpha}=0$.

But one can hardly justify energy conservation by any physical argument. In fact the deformation induced by the impact is expected to propagate dynamically all through the various parts of the system and possibly also through the external connected bodies. Even if the (very slightly) deformable 
materials of which the system is built may be asserted perfectly elastic, the various parts usually remain, after a bounce, in a state of vibration which, in the energy balance drawn at macroscopic level, amounts to dissipation.

In short, predicting accurately the outcome of a shock requires some higher order information, unavallable in usual situations. The pertinence of the model of an elastic shock has to be discussed in each particular application. The same is true for the other sort of shock. we shall present in Sec.8, which however of fers the advantage of better formal consistency and easier numerical handling.

\section{NONSMOOTHDYNAMICS}

We now intend to insert the description of shocks into a generalized formulation of the dynamics of the investigated system, which does not require the local absolute continuity of the velocity function $u: l \rightarrow \mathbb{R}^{n}$. This function will only be assumed to have locally bounded variation, i.e. to have bounded variation on every compact subinterval of 1 ; notation: $u \in \operatorname{Ibv}\left(I, \mathbb{R}^{n}\right)$. Classically, with such a function, an $\mathbb{R}^{n}$-valued measure on the interval $I$ is associated, that we shall call the differential measure of $u$ and denote by du. A characteristic property of this measure is that, for every compact subinterval $[\sigma, \tau] \subset$ int 1 , one has

$$
\int_{[\sigma, \tau]} d u=u^{+}(\tau)-u^{-}(\sigma)
$$

In fact, the bounded variation assumption secures the existence of the one-sided limits of $u$ at any point of int I. Equality (7.1) remains valid for $\sigma=t_{0}$ provided that, as already proposed in Sec. 2 , we agree on the convention 
$u^{-}\left(t_{0}\right)=u\left(t_{0}\right)$. The symmetrical convention may also be used for $u^{+}$at the possible right end of 1 .

By making $\sigma=\tau$, one sees in particular that, if $u$ is discontinuous at point $\tau$, then the measure $d u$ is expected to possess an atom at this point, with value equal to the total jump of $u$.

It is clear on (7.1) that du depends on the function $u$ only through $\mathrm{u}^{+}$ and $\mathrm{u}^{-}$. The values that $\mathrm{u}$ may take at its discont inuity points are immaterial. Neither have these values any efrect on the expression (2.1) of a since the set of the discontinuity points or $u$ is countable, hence. Lebesque-negligible.

The reader may refer to [7] as a monograph on the lbv functions of a real interval and their differential measures, with values in a Banach space $X$. For a vast class of Banach spaces, in particular for $X=\mathbb{R}^{n}$, there comes out that, if $u$ is locally absolutely continuous, the measure du possesses, relatively to the Lebesgue measure on 1 , here denoted by $\mathrm{dt}$, a density runction, say $u_{t}^{\prime} \in \mathcal{L}_{\text {loc }}^{1}\left(l, d t ; \mathbb{R}^{n}\right)$. Of course, the latter is defined up to the addition or a dt-negligible function. This is commonly expressed by saying that the $\mathbb{R}^{n}$-valued measure du equals the product of the real measure dt by the $\mathbb{R}^{n}$ - valued, locally dt-integrable, function $u_{t}^{\prime}$; notation $d u=u_{t}^{\prime} d t$. Also du is said locally dt-continuous. Conversely, if du has such a form, the function $u \in \mid \operatorname{bv}\left(I, \mathbb{R}^{n}\right)$, possibly after correcting the unessential values it takes on a countable set, is locally absolutely continuous. Furthermore, for Lebesgue-almost every $t$, the value $u_{t}^{\prime}(t)$ of the density function makes the (two-sided) derivative of $u$.

Throughout these lectures, we shall comply with the widespread usage 
of affecting the character $\mathcal{L}$ to non-Hausdorff spaces consisting of functions defined everywhere, while $L$ will refer to the corresponding Hausdorff spaces of equivalence classes.

In this setting, let us come back to Lagrange equations. If $u$ is locally absolutely continuous, with a related to it through (2.1), the notation used in Sec. 4 becomes

$$
A_{i j}(q) u_{i}^{j}+\left(A_{i j, k}(q)-\frac{1}{2} A_{j k, i}(q)\right) u^{j} u^{k}=c_{i j}
$$

The right-hand side refers to the covariant components of the totality of the forces acting on the system, including the reactions of possible contacts. For these differential equations to make sense, the $n$ functions $t \rightarrow c_{i}$ have to be elements of $\mathcal{L}^{\prime}{ }_{\text {loc }}(1, \mathrm{dt} ; \mathbb{R})$; so each of the $n$ equations (7,2) may equivalently be expressed as an equality of measures on the interval I,

$$
A_{i j}(q) d u^{j}+\left(A_{i j, k}(q)-\frac{1}{2} A_{j k, i}(q)\right) u^{j} u^{k} d t=c_{i} d t .
$$

One readily checks that the functions of $t$, by which the measures $\mathrm{du}^{\mathrm{j}}$ or $\mathrm{dt}$, on the left-hand side, are multiplied, have the local integrability properties required in order that the products make sense.

Now, this new writing keeps meaningful for general $u \in \mid b v\left(1, \mathbb{R}^{n}\right)$, and allows one to replace the terms $c_{j} \mathrm{dt}$, on the right-hand side by some real measures $\mathrm{dC}_{j}$, said to be the covariant components of the total impulsion $\mathrm{dC}$ experienced by the system. These will equal the sum of the dt-continuous measures $F_{i}(t, q, u) d t$, describing given forces, and of the covariant components $\mathrm{dR}_{\mathrm{j}}$ of the contact impulsion $\mathrm{dR}$, an $\mathbb{R}^{n}$-valued measure on I. For instance, in the case of an isolated collision occuring at instant $t_{s}$, as 
investigated in $\operatorname{Sec} .6$, the measure $d R$ would involve an atom with mass $\pi$, placed at point $t_{s}$; this should be added to the dt-continuous measure $r \mathrm{dt}$, expressing the contact actions in the course of possible episodes of smooth motion with persistent contact.

As before, we shall denote by $K$ the known $\mathbb{R}^{n}$-valued function with components

$$
K_{i}(t, q, u)=F_{i}(t, q, u)-\left(A_{i j, k}(q)-\frac{1}{2} A_{j k, i}(q)\right) u^{j} u^{k} .
$$

Then the rollowing equality of $\mathbb{R}^{n}$ - valued measures on the interval 1 will be adopted as governing the dynamics of possibly nonsmooth motions:

$$
A(q) d u-K(t, q, u) d t=d R .
$$

The connection between such an extension of Lagrange equations and the principles of Classical Dynamics is discussed with more precision in [9] .

\section{CONTACT SOFTNESS}

Let us consider again the differential inclusion (5.6), which has been found to govern the assumedly smooth motions of the system. This inclusion equivalently means that the expression $t \rightarrow r(t) \in \mathbb{R}^{n}$ of the covariant components of the contact forces makes an element of $\mathcal{L}^{1}{ }_{\text {loc }}\left(1, \mathrm{dt}, \mathbb{R}^{n}\right)$ which verifies, for Lebesgue-almost every $t$,

$$
-r(t) \in \partial \psi_{V(q(t))}(u(t))
$$

In the context of Sec.5, $q(t)$ belongs to $\Phi$ for every $t$; therefore, due to Prop. 2.2 and to the continuity of $u$, one has $u(t) \in V(q(t))$ for every t. Hence, the right-hand side of $(8.1)$ is nonempty for every $t$ (it contains at least the 
zero of $\mathbb{R}^{n}$ ). This enables one, by altering the function $r$ on a Lebesguenegligible subset of 1 , to make (8.1) hold everywhere in 1 .

In the language of $\operatorname{Sec} .7$, the $\mathbb{R}^{n}$-valued measure $d R=r d t$ constitutes, for the above case, the total contact impulsion. We now propose to adapt (8.1) to the more general setting of Nonsmooth Dynamics.

The Lebesgue measure thus loses its preeminence and we shall definitely give up using the expression "almost everywhere". As observed in Sec.7, if $u$ is discontinuous, only $u^{+}$and $u^{-}$have relevance to the motion; so, one has to make a choice about what is to replace $u$ in the right-hand side of (8.1).

DEFINITION 8.1 The set of superimposed constraints is said frictionless and soft if the total contact impulsion admits a representation $\mathrm{dR}=\mathrm{R}_{\mathrm{U}}^{\prime} \mathrm{d} \boldsymbol{U}$, where du denotes a nonnegative real measure on 1 and $R_{u}$ an element or $\mathcal{L}^{1}{ }_{100}\left(1, \mathrm{du} ; \mathbb{R}^{\mathrm{n}}\right)$ such that, for every $\mathrm{t}$ in 1 .

$$
-R^{\prime}(t) \in \partial \psi_{v(q(t))}\left(u^{+}(t)\right)
$$

This first imples that the right-hand side is nonempty, hence $u^{+}(t) \in V(q(t))$ for every $t$. Therefore, if the initial data satisfy $q\left(t_{0}\right) \in \Phi,(8.2)$ will secure, thanks to Prop. 2.4, that $q(t) \notin \Phi$ for every $t \in l$.

The following Proposition shows that the concept introduced by Definition 8.1 does not actually depend on the peculiar choice of $d u$.

PROPOSITION 8.2 inclusion (8.2) holds for every $t \in$ if and only if the same is true after replacing dy by another nonnegative real measure relative to which $\mathrm{dR}$ possesses a density function. 
PROOF Suppose that (8.2) holds for every $t$ in I and denote by $d v$ another nonnegative real measure such that $R_{v}^{\prime}$ exists. If we put $d \sigma=d \mu+d v$, the Radon-Nikodym theorem ensures the existence of the density functions $\varphi_{\mathfrak{o}}$ and $v_{\sigma}^{\prime}$, nonnegative elements of $\mathcal{L}^{\infty}(l, d \sigma ; R)$. Then $d R=R_{\mu}^{\prime} \mu_{\sigma}^{\prime} d \sigma=R_{\nu}^{\prime} \nu_{\sigma}^{\prime} d \sigma$; so the $\mathbb{R}^{n}$-valued functions $R_{\mu}^{\prime} \mu_{\sigma}^{\prime}$ and $R_{\nu}^{\prime} v_{\sigma}^{\prime}$ are equal, except possibly in some $d \sigma$-negligible (hence $d v$-negligible) subset $S$ of 1 . The subset $N=\{t \in \mid$ : $\left.v_{d}^{\prime}(t)=0\right\}$ is $d v$-negligible. Outside the union SUJN, the above implies $R_{v}^{\prime}=$ $\mathrm{R}_{\mu}^{\prime} \mu_{\sigma}^{\prime} / v_{\sigma}^{\prime}$, with $\mu_{\sigma}^{\prime} / \nu_{\sigma}^{\prime} \geqslant 0$; then the expected inclusion holds, since the righthand member of (8.2) is a cone. For $t \in S \cup N$, this (closed, convex) cone, being nonempty, contains at least the zero of $\mathbb{R}^{n}$. After replacing by zero the values that the function $R_{v}^{\prime}$, as formerly defined, may take in SUN, one obtains the asserted conclusion, with dy and dv playing symmetric roles.

If the superimposed constraints agree with Definition 8.1 , the elimination of $d R$ between (8.2) and the equation (7.4) of Nonsmooth Dynamics yields the following characterization of the possible motions

$$
-A(q) u_{\psi}^{\prime}+k(t, q, u) t^{\prime} \in \partial \psi_{v(q(t))}\left(u^{+}(t)\right)
$$

required to hold for every $t \in l$. Here $d \mu$ may equivalently be replaced by any nonnegative real measure, relative to which du and dt possess density functions. The existence of such measures is a priori secured by the Radon-Nikodym theorem; one may take, for instance, $d y=|d u|+d t$, where ldul denotes the nonnegative real measure modulus (or variation measure) [6][7] of the vector measure du. Since $K$ is continuous and since the discontinuity set of $u$ is $\mathrm{dt}$-negligible, it does no matter to replace $u$, on the left-hand side, by $\mathrm{u}^{+}$or $\mathrm{u}^{-}$. 
The indifference of (8.3) regarding the choice of $d y$ (this could be checked directly, through the same reasoning as in the proof of Prop. 8.2), suggests to strip down the writing into

$$
-A(q) d u+K(t, q, u) d t \in \partial \psi_{v(q(t))}\left(u^{+}(t)\right) .
$$

This may be called a measure differential inclusion. The existence of solutions to initial value problems governed by conditions of this sort and their possible uniqueness have so far been studied only in some special cases $[11][12][13][31]$.

As before, one observes that, provided that $q\left(t_{0}\right) \in \Phi$, inclusion (8.3) entails $q(t) \in \Phi$ for every $t \in l$.

REMARK 8.3 Similarly to what precedes, the simple assumption of no-friction, without reference to "softness", as it has been expressed for the case of smooth motions in (5.3), may be adapted to Nonsmooth Dynamics. It will merely consist in stating that the contact impulsion dR possesses, relative to some $d y \geqslant 0$, a density function which satisfies for every $t \in 1$

$$
-R_{U}^{\prime}(t) \in N(q(t)) \text {. }
$$

Here again, the fact that the right-hand member is a cone entails that such an assumption does not actually depend on the peculiar choice of dy.

since the right-hand member of (8.2) is contained in $N(q(t)),(8.5)$ constitutes a weaker assumption than what expresses Definition 8.1, 1.e. the set of the superimposed constraints may be frictionless without being soft.

REMARK 8.4 Classically, a subdifferential relation such as (8.2) admits some alternative formulations. Here, since the polar cone of $V(q)$ equals $N(q)$, (8.2) is found equivalent to asserting, for every $t$, 


$$
u^{+} \in V(q), \quad-R_{u}^{\prime} \in N(q), \quad u^{+} \cdot R_{\mu}^{\prime}=0
$$

\section{SOFT COLLISIONS}

In the setting of the above Section, the following characterizes the possible velocity jumps.

PROPOSITION 9.1 Let $\tau \in \mathrm{I}$, different from the possible right end of this interval. For any motion satisfying (8.3), one has

$$
u^{+}(\tau)=\operatorname{prox}\left(u^{-}(\tau), v(q(\tau)),\right.
$$

where the proximation is understood in the sense of the kinetic metric, i.e. the Euclidean metric defined in $\mathbb{R}^{n}$ by the matrix $A(q(\tau))$

PROOF In View of (7.1),

$$
u^{+}(\tau)-u^{-}(\tau)=\int_{\{\tau\}} d u=\int_{\{\tau\}} u^{u^{\prime}} d u=u_{\tau} u_{u}^{\prime}(\tau)
$$

where $\mu_{\tau} \geqslant 0$ denotes the integral of $d \mu$ over the singleton $\{\tau$. If motion agrees with (8.3), one has

$$
-A(q(\tau)) u_{\psi^{\prime}}(\tau)+K(\tau, q(\tau), u(\tau)) t_{\psi^{\prime}}(\tau) \in \partial \psi_{v(q(\tau))}\left(u^{+}(\tau)\right) .
$$

Now $\psi_{\tau} t_{v}^{\prime}(\tau)=0$, since the Lebesgue measure at has no atom. Then, after multiplying both members of the above by $\mu_{\tau}$, one obtains

$$
-A(q(\tau))\left(u^{+}(\tau)-u^{-}(\tau)\right) \in \partial \psi_{v(q(\tau))}\left(u^{+}(\tau)\right) .
$$

If one uses in $\mathbb{R}^{n}$ a base orthonormal relatively to the metric in view, $A(q(\tau)$ ) becomes the unit matrix, reducing this to the classical characteristic property of proximal points.

This Proposition, which, under the convention $u^{-}\left(t_{0}\right)=u\left(t_{0}\right)$, also holds 
for $\tau=\mathrm{t}_{0}$, shows that $u$ exhibits a nonzero jump at point $\tau$ if and only if $U^{\top}(\tau) \notin V(q(\tau))$, i.e. a nontangential impact occurs at this instant.

Assume in addition that $\tau>\tau_{0}$, so $u^{-}(\tau) \epsilon-V(q(\tau))$ in view of Prop.2.2. Then, the condition for nonzero velocity jump becomes

$$
U^{-}(\tau) \in V(q(\tau)) \cap-V(q(\tau))
$$

The right-hand member is a linear subspace of $\mathbb{R}^{n}$. If $J(q(\tau))=\varnothing$, i.e. $q(\tau) \in$ int $\Phi$, this is the whole of $\mathbb{R}^{n}$. If $J(q(\tau))$ consists of a singleton, say $\{\alpha\}$, the subspace is the vector hyperplane tangent at $q(\tau)$ to hypersurface $f_{\alpha}=$ const.. For larger $J(q(\tau))$, the point $q(\tau)$ lies on what may be called, in the wide sense, an edge and the said subspace (possibly reduced to $\{0\}$ ) is declared tangent to this edge.

Also for $\tau>\tau_{0}$, one observes that $u^{-}(\tau)$, being an element of $-V(q(t))$, cannot belong to the interior of $V(q(\tau))$; thus $(9.1)$ yields that $u^{+}$actually lies on the boundary of this polyhedral cone.

REMARK 9.2 Here is another consequence of Prop.9.1. Suppose that, on some open subinterval l' of 1 , the motion evolves in such a way that the set-valued function $t \rightarrow J(q(t))$ never increases. In other words, during this time interval, some of the contacts may get loose, but no collision occurs. Let $\tau \in l^{\prime}$ and let $\alpha \in J(q(\tau))$. Then, for every $t \in l^{\prime}$ such that $t \leqslant \tau$, one has $f_{\alpha}(q(t))=0$, an equality which, through the chain rule, entails

$$
u^{-}(\tau) \cdot \nabla f_{\alpha}(q(\tau))=0
$$

Hence $U^{-}(\tau) \in V(q(\tau))$, so Prop.9.1 shows that no velocity jump occurs at instant $\tau$.

In the traditional treatment of unilateral constraints, the latter is taken for granted: one accepts to enter percussions into the analysis only at 
instants where geometry makes them unavoidable. This agrees with the heuristic maxim of the "minimal singularity", but does not result from any explicit mechanical assumption. Here is a familiar example demonstrating this method deficiency.

Suppose an object performing a sliding motion in the contact of a table (or simply at rest on it). If an operator hits the table with a hammer, the object is commonly observed to jump. So the table has imparted an impulse to the contacting object, without itself exhibiting any motion at the macroscopic observation scale. In contrast, the assumption that unilateral constraints are frictionless and soft rules out such an active behaviour of boundaries.

Incidentally, the replacement of softness, in frictionless unilateral constraints, by the quite different assumption of energy conservation would also permit a deductive treatment of the above situation. It will be shown in Sec.10 that energy loss, in velocity jumps, should on the contrary be expected when frictionless soft constraints are present.

REMARK 9.3 Equation (9.1) expresses that, in the considered motion, all velocity jumps are of the sort that the author has previoulsy called standard inelastic shocks [14][15]. These were proposed as a generalization of the shocks which, in the case of a system involving a single constraint inequality, say $f(q) \leqslant 0$, are traditionally called "inelastic" or also "soft". In fact, if $f\left(q\left(t_{s}\right)\right)=0$, the tangent cone to the feasible region $\Phi$ of $\mathbb{R}^{n}$ at point $q_{s}=q\left(t_{s}\right)$ is simply the half-space $v\left(q_{s}\right)=\left\{v \in \mathbb{R}^{n}: v \cdot \nabla f\left(q_{s}\right) \leqslant 0\right\}$. Since the left-side velocity $u^{-}\left(t_{s}\right)$ must belong to $-V\left(q_{s}\right)$ (at least if one supposes $\left.t_{s}>t_{0}\right)$, equ.(9.1) ylelds in this special case that $u^{+}\left(t_{s}\right)$ equals the orthogo- 
nal projection of $\mathrm{u}^{-}\left(\mathrm{t}_{\mathrm{s}}\right)$ to the vector hyperplane tangent at $\mathrm{a}_{\mathrm{s}}$ to hypersurface $\mathrm{f}=0$. More information on general standard inelastic shocks may be found in [15] ; incidentally, the contact percussion receives an extremal characterization, dual to (9.1).

\section{ENERGY BALANCE}

PROPOSITION 10.1 For every motion satisfying (8.3) the function $t \rightarrow \mathcal{E}_{\mathrm{c}}$ belongs to $I \mathrm{bv}(I, \mathbb{R})$. In the sense of the ordering of real measures on 1 , one has

$$
\mathrm{d} \mathbf{E}_{\mathrm{c}} \leqslant \mathrm{F}, \mathrm{dq},
$$

with equality if and only if $\mathrm{u}$ has no jump in 1 .

PROOF For the traditional case of smooth motions w/th frictionless (time-independent) superimposed constraints, the power equation

$$
\frac{\mathrm{d}}{\mathrm{dt}} \mathcal{E}_{\mathrm{c}}=\mathrm{F}_{\mathrm{i}}(\mathrm{t}, \mathrm{q}, \mathrm{u}) \mathrm{u}^{\mathrm{t}}
$$

is easily derived from Lagrange equations, with left-hand side developed in the form (4.3). For a motion governed by (7.4), with the contact impulsion $\alpha R=R_{u}^{\prime} d u$ satisfying (8.2), there is only to retrace the same calculation, under the replacement of some steps, based on the rules of usual Differential Calculus, by what follows.

$1^{\circ}$ If $u^{j}$ and $u^{j}$ belong to $\operatorname{lbv}(1, \mathbb{R})$, the same holds for the product $u^{i} u^{j}$ and its differential measure is given [7] [32] by

$$
d\left(u^{j} u^{j}\right)=u^{i+} d u^{j}+u^{j-} d u^{j} \text {. }
$$

The products of measures by functions, which appear on the right-hand side, make sense because the functions $t \rightarrow u^{i+}(t)$ and $t \rightarrow u^{j-}$ belong to $l b v(I, \mathbb{R})$; so they are locally integrable relative to any real measure. Hence, in view of the symmetry of $A$, 


$$
A_{i j}(q) d\left(u^{j} u^{j}\right)=A_{i j}(q)\left(u^{l+}+u^{i-}\right) d u^{j}
$$

Furthermore, since $t \rightarrow q(t)$ is locally absolutely continuous, with $t \rightarrow u(t)$ as derivative, the differential measure of $t \rightarrow A_{i j}(q(t))$ equals

$$
d A_{i j}=A_{i j, k} u^{k} d t
$$

and one has

$$
d\left(A_{i j} u^{i} u^{j}\right)=u^{i} u^{j} d A_{i j}+A_{i j} d\left(u^{i} u^{j}\right)
$$

$2^{\circ}$ The real measure $A_{i j}(q)\left(u^{i+}-u^{i-}\right) d u^{j}$ is nonnegative; it vanishes if and only if $u$ is continuous on 1 . In fact, this measure consists of a countable and locally summable collection of point measures located at the jump instants of $u$. Let $t_{s}$ denote one of these instants; under the notations $u^{+}\left(t_{s}\right)=u_{s}^{+}, u^{-}\left(t_{s}\right)=u_{s}^{-}, q\left(t_{s}\right)=a_{s}$, the mass of the corresponding point measure equals

$$
A_{i j}\left(q_{s}\right)\left(u_{s}^{i+}-u_{s}^{i-}\right)\left(u_{s}^{j+}-u_{s}^{j-}\right) .
$$

Since $A_{i j}(q)$ is, for every $q$, a positive definite matrix, this real number is nonnegative; it vanishes if and only if $u_{s}^{+}-u_{s}^{-}=0$.

$3^{\circ}$ Equality u. $r=0$, a consequence of the no-friction assumption in the case of smooth motions, is replaced at present by $u^{+} \cdot R_{u^{\prime}}=0$, a fact observed in (8.6).

REMARK 10.2 A more general concept than frictionless soft constraints is obtained by inserting into the right-hand side of (8.2), instead of $\mathrm{u}^{+}$, some weighted mean

$$
u_{6}=\frac{1}{2}(1+\delta) u^{+}+\frac{1}{2}(1-\delta) u^{-},
$$

where $\sigma$ is a chosen real number, here supposed independent of $t$, for simplicity. Since $u_{g}(t)=u(t)$, except at the jump points of $u$, which make a 
Lebesgue-negligible subset of I, the law of constraint

$$
-R_{u}^{\prime}(t) \in \partial \psi_{v(q(t))}\left(u_{\delta}(t)\right)
$$

implies, exactly like (8.2), that condition $q(t) \in \Phi$ is satisfied for every $t$ as soon as it holds for $\mathrm{t}=\mathrm{t}_{0}$

For $6>0$ (even larger than 1), the law of constraint (10.3) entails the same as what has been stated in Prop.10.1. Choosing $\delta<0$ would yield the reverse inequality, physically unacceptable (unless the possible bounces are artificially enhanced, as in some electric billiard games).

If $\delta=0$, i.e. $u_{\delta}$ equals the midpoint of $u^{-}$and $u^{+}$, one finds equality $d \mathcal{E}_{\mathrm{r}}=\mathrm{F} . \mathrm{dq}$, expressing the same energy conservation as in smooth motions. In that sense, the constraint law (10.3) with $\delta=0$ may be said "elastic".

We suggest to call $\delta$ the dissipation index of the constraint law (10.3).

\section{TIME DISCRETIZATION ALGORITHM}

The principle of such an algorithm has been introduced, for smooth motions, in Remark 5.3. The main observation we now have to make is that the same numerical technique applies in the framework of Nonsmooth Dynamics, provided that the superimposed frictionless unilateral constraints are assumed soft.

The expression in (7.4) of the system dynamics, in term of measures, directly suggests time discretization: the rule will be to equal some approximants of the respective integrals of both members over each subinterval of I determined by the discretization nodes.

Let $\left(t_{1}, t_{F}\right)$ be one of these intervals (here I refers to "initial" an $F$ to "f inal"), with length $h=t_{F}-t_{1}$ possibly variable from one step to another. From the approximants $q_{1}$ and $u_{1}$ of $q$ and $u$ at the beginning of the interval, one has 
to compute some approximants $a_{F}$ and $u_{F}$, assigned to instant $t_{F}$ and which, in turn, will be used as initial values in the next step.

Stage 1. Calculate $t_{M}=t_{1}+\frac{1}{2} h$ and some midooint approximants

$$
q_{M}=a_{1}+\frac{1}{2} n u_{1} \in \mathbb{R}^{n}, \quad A_{M}=A\left(a_{M}\right) \in \mathbb{R}^{n \times n}, \quad K_{M}=k\left(t_{M}, q_{M}, u_{1}\right) \in \mathbb{R}^{n} .
$$

Then

$$
u_{L}=u_{1}+h A_{M}^{-1} K_{M}
$$

is the value that the discretized equations of Dynamics would yield for $u_{F}$ in the absence of contact force (here one may read the subscript $L$ as referring to "loose").

If $q_{M} \in$ int $\Phi$ (i.e. all $f_{\alpha}\left(q_{M}\right)$ are strictly negative) or $u_{L} \in V\left(q_{M}\right)$, one considers that contact forces have no effect on the calculated step; so one makes $u_{F}=u_{L}$ and goes to Stage 3 (this decision is actually a trivial case of stage 2 below).

Stage 2. On the left-hand side of (8.4), let us replace $A(q)$ and $K(t, q, u)$ by $A_{M-1}$ and $K_{M}$. Then, an approximant of the integral of this left-hand side over $\left(t_{1}, t_{F}\right)$ is $-A_{M}\left(u_{F}-u_{L}\right)$. Concerning the right-hand side, it will be considered that the set $V(q(t))$ keeps, throughout the interval, the constant value $V\left(a_{M}\right)$, whose indicator function will be denoted by $\psi_{M}$. Furthermore, let us take $u_{F}$ as an approximant-one may rather say a simulation- of $u^{+}$. This yields as a simulation of (8.4) on the said interval

$$
-A_{M}\left(u_{F}-u_{L}\right) \in \partial \psi_{M}\left(u_{F}\right)
$$

If the matrix $A_{M}$ is used in order to def ine a Euclidean metric on $\mathbb{R}^{n}$, this characterizes $u_{F}$ as the proximal point to $u_{L}$ in the closed convex set $v\left(q_{M}\right)$. Therefore, computing $u_{F}$ is a Quadratic Programming problem: to minimize 
on $V\left(q_{M}\right)$ the real function $x \rightarrow\left(x-u_{L}\right) \cdot A_{M}\left(x-u_{L}\right)$. Recall that $V\left(q_{M}\right)$ is a polyhedral cone, the intersection of a collection of half-spaces determined by the index set $J\left(q_{M}\right)$. These correspond to the values of $\alpha$ such that, in the test position $q_{M}$, the inequality $f_{\alpha} \leqslant 0$ holds as an equality or is violated. If their number is not too large, the proximal point will be constructed algebraically; otherwise some of the classical algorithms of Quadratic Programming will have to be applied.

Stage 3. One terminates the computation step by

$$
a_{F}=a_{M}+\frac{1}{2} n u_{F} \text {. }
$$

REMARK 11.1 In [24] are reported some computer experiments with methods of the above sort. These methods prove to be stable. The finer the time discretization is, the better the computed motion complies with the inequalities $f_{\alpha} \leqslant 0$. In that respect, some improvement may be achieved by evaluating, in Stage 2, the cone $V(q)$ at another point than $q_{M}$. A good choice appears to be the point $q_{1}+h u_{1}$.

If these methods are applied to the calculation of mechanisms, which in reality always involve some imperfectly known friction, no great precision can anyway be expected in predicting the motion. From that viewpoint, a moderately fine time discretization will be enough. In contrast, the violation of the constraint inequalities must sharply be kept in check. A very effective way of doing it consists in completing each time step by a stage of linear correction of the possible violation. Let us explain it in the simple case where the position $a_{F}$, calculated in Stage 3 , violates only one of the constraint inequalities. In other words, the real number $\varphi=f\left(q_{F}\right)$ is found strictly positive, with $f$ denoting one of the functions $f_{\alpha}$. A plausible correction of 
this violation (naturally assumed "small") would be to replace a by its proximal point, say $q_{c}$, in the region $f \leqslant 0$. Proximity here should be understood in the sense of some Euclidean metric on $\mathbb{R}^{n}$; the most justified choice is to rely on the kinetic metric, defined by the matrix $A(q)$. To save computation, the latter will be evaluated at a point where it has already been calculated in the current step. Using an affine approximation of the function $f$, one obtains $0=f\left(q_{C}\right)=\varphi+\left(q_{C}-q_{F}\right) . \nabla f+$. . Here the gradient $\nabla f$ should be evaluated at some neighbour point. This precisely must have been done at the stage 2 of the current step, where also the vector $A^{-1} \nabla f$ has been needed. Defining $a_{C}$ as the proximal point to $q_{F}$ in the region $i \leqslant 0$, relative to the metric in view, means that the vector $a_{C}-q_{F}$ is parallei to $A^{-1} \nabla f\left(q_{C}\right)$. Since the latter is estimated to be close to the calculated value of $A^{-1} \nabla f$, this finally yields the approximate formula

$$
a_{C}=a_{F}-\varphi\left(\nabla f \cdot A^{-1} \nabla f\right)^{-1} A^{-1} \nabla f+\ldots
$$

The same techniques of violation control applies to the numerical methods presented in the sequel, for unilateral constraints with friction.

REMARK 11.2 Also in [24], a numerical procedure is developed for a system with a frictionless unilateral constraint expressed by a single inequality $f \leqslant 0$, assuming that the dissipation index, as we have defined it in Sec.10, equals 1 . In other words, the possible collisions are elastic bounces. In contrast with the excellent stabllity of the preceding method, a careful check of the energy balance of each time-step here is needed, in order to prevent divergent oscillations when the algorithm is applied to the computation of a motion with assumedly persistent contact. 


\section{COULOMB FRICTION}

This lectures are meant to provide only an introduction to the treatment of friction. So we shall restrict ourselves to a system involving a single unilateral constraint with geometric condition $f(q) \leqslant 0$ (there is presented in [30] a rather usual case, where a system with multiple possible contacts may be reduced, through decomposition, to this simple setting).

For every $q$ such that $f(q) \geqslant 0$, the cone $V(q)$ equals the half-space $\left\{v \in \mathbb{R}^{n}\right.$ : $\vee \nabla f(q) \leqslant 0\}$, with boundary

$$
T(q)=\left\{v \in \mathbb{R}^{n}: v \cdot \nabla f(q)=0\right\}
$$

the latter is the vector hyperplane tangent at point $q$ to the hypersurface $f=$ const. drawn through this point.

Let a motion satisfy $f(q(t)) \leqslant 0$ for every $t \in I$. If the velocity function $u$ is continuous at some $\tau>t_{0}$, then, in view of Prop.2.2, $u(\tau)$ belongs to $V(q(\tau)) \cap-V(q(\tau))$; this set equals $T(q(\tau))$ if $f(q(\tau)) \geqslant 0$ and otherwise the whole of $\mathbb{R}^{n}$. The same is true for $t=t_{0}$, as far as the initial data have the meaning we agreed to give them in Sect.2, namely $u_{0}$ equals the left-limit $u^{-}\left(t_{0}\right)$ in a motion taking place before $t_{0}$, with the unilateral constraint already in effect.

To $f i x$ the ideas, suppose, as in $\operatorname{Sec} 3$, that equality $f(q)=0$ expresses that in the position q, some part $\mathcal{B}_{1}$ of the system touches the unmoving external obstacle $\mathcal{B}_{0}$. Then, under the previous notations,

$$
U=V\left(\mathcal{M}_{1}(q), q, u\right)=G_{q} u
$$

is the velocity of $\mathscr{B}_{1}$ relative to $\mathcal{B}_{0}$ at the contact point.

Denoting again by $\mathcal{N}_{\mathrm{q}}$ the common normal unit vector to the contacting bodies, directed toward $\mathcal{B}_{1}$, we assume $\mathcal{G}_{q}^{*} \mathcal{N}_{q} \neq 0$ (see Remark 3.2). Then the assertion $u \in T(q)$ is equivalent to $U$ belonging to $T(q)$, the linear subspace of 
$\mathcal{E}_{3}$ orthogonal to $\mathcal{N}_{q}$, le. the common tangent vector plane at the contact point.

The above is the situation in which, traditionally, Coulomb's law of dry friction is formulated. This law is a relation between the sliding velocity $U \in T(q)$ and the contact force $R \in \mathcal{F}_{3}$ experienced by $\mathcal{B}_{1}$

For brevity, let us write $\mathcal{N}$ for $\mathcal{N}_{q}$ and $\mathcal{T}$ for $\mathcal{T}(q)$. The familiar formulation rests on the decomposition of $R$ into

$$
R=R_{T}+\rho \mathcal{N} \text {, with } R_{T} \in \mathcal{T} \text { and } \rho \geq 0 \text {, }
$$

and consists of two well known separate assertions concerning the respective cases $U=0$ and $U_{\neq} 0$ in $\tau$.

In some of the author's early papers [20] [21], it has been observed that, as far as the normal component $\rho$ is treated as known, this pair of assertions is equivalent to a relation, between the elements $R_{T}$ and $u$ of the linear space $\tau$, which derives from a "pseudo-potential". Furthermore, this formulation readily extends to the description of possibly anisotropic friction, as a relation of the form

$$
-u \in \partial \psi_{\mathcal{D}}\left(\mathcal{R}_{T}\right)
$$

with $\mathcal{D}=\varrho \mathcal{D}_{1}$. By $\mathcal{D}_{1}$ is denoted a given closed convex subset of $\mathcal{T}$, containing the origin; in the traditional case of isotropic friction, $\mathcal{D}_{1}$ equals the disk centered at the origin, with radius equal to the friction coefficient, say $y$.

we shall restrict ourselves in these lectures to the case of bounded friction, i.e the set $\mathcal{D}_{1}$ is bounded. To take $\mathcal{D}_{1}$ unbounded would provide a way of including in our approach the situation traditionally called a nonholonomic constraint. But this would cause some complications in further statements.

Through the standard calculation rules of Convex Analysis, (12.2) may 
equivalently be written as

$$
-R_{T} \in \partial \varphi(u)
$$

where the dissipation function $\varphi$ equals the support function of the set $-\mathcal{D}_{1}$; in particular, for isotropic friction $\varphi=\gamma\|l\|$.

Elementary applications, where $\rho$ in fact is known, may be found in [22]. Furthermore, having to treat $\rho$ as known does not prevent using this pseudo-potentlal formulation (or an equivalent variational inequality which expresses a "principle of maximal dissipation") in the proof of existence of solutions to dynamical problems. See [33], where the normal components of the contact forces become the primary unknowns in some functional analytic arguments

In the present lectures, we choose to formulate the same law under a conical equivalent form [24], avoiding the decomposition (12.1). The friction cone $\mathrm{C}$ at the contact point is introduced, a closed convex conical subset of the linear space $\mathcal{E}_{3}$ (recall that, speaking of a cone in a linear space, one understands that it has vertex at the origin). In traditional isotropic friction, this is a cone of revolution about $\mathcal{N}$; generally, $C$ equals the cone generated in $\mathcal{E}_{3}$ by the set $\mathcal{D}_{1}+\mathcal{N}$. So $\mathcal{C}$ contains $\mathcal{N}$ and lies entirely on the corresponding side of $T$.

PROPOSITION 12.1 The pair of relations $(12.1),(12.2)$ is equivalent to

$$
-u \in \operatorname{proj}_{\mathcal{T}} \partial \Psi_{C}(\mathcal{R})
$$

PROOF Assume that (12.4) holds; hence $-\mathcal{U} \in \mathcal{T}$. Derioting by $[\mathcal{N}]$ the linear subspace generated in $\mathcal{E}_{3}$ by $\mathcal{N}$, one has

$$
\exists V \in \partial \Psi_{C}(R), \exists W \in[\mathcal{N}]:-U=v+W
$$

Let us decompose $R$ in the form (12.1); necessarily $\rho \geqslant 0$, since (12.4) implies 
that $\partial \psi_{C}(R)$ is nonempty, thus $R \in C$. By construction, $R$ belongs to the affine plane $\mathcal{A}=\mathcal{T}+\rho \mathcal{N}$. Therefore $\partial_{\mathcal{A}}(\mathcal{R})=[\mathcal{N}]$ and (12.5) means that

$$
-U \in \partial \psi_{\mathcal{A}}(\mathcal{R})+\partial \psi_{C}(\mathcal{R}) \subset \partial \psi_{\mathcal{A}} \cap \mathrm{C}^{(\mathcal{R})}
$$

In view of the definition of $\mathcal{D}$, one has $\mathcal{A} \cap \mathcal{C}=\mathcal{D}+\varrho \mathcal{N}$, thus, using a translation in the evaluation of subdiffentials,

$$
{ }^{\partial} \psi_{A} \cap C^{(\mathcal{R})}=\partial \psi_{\mathcal{D}}(\mathcal{R}-\rho \mathcal{N})=\partial \psi_{\mathcal{D}}\left(\mathcal{R}_{\mathrm{T}}\right)
$$

Then (12.6) entails that (12.2) holds in the sense of the Euclidean autoduality of $\mathcal{E}_{3}$. Since $U$ and $\mathcal{R}_{\mathrm{T}}$ are elements of the linear subspace $T$, the same is true relatively to the Euclidean autoduality of this subspace.

Conversely, let us assume that $U \in T$ and that (12.2) holds in the sense of the Euclidean autoduality of $\mathcal{T}$, with $\mathcal{R}_{T}$ defined by $(12.1)$ (observe that $\rho \geqslant 0$ is stated at this place). Then (12.2) is true also in the sense of the autoduality of $\mathcal{E}_{3}$ and, using translations as above, one concludes

$$
-u \in \partial \psi_{\mathcal{A}} \mathcal{C}^{(\mathcal{R})} \text {. }
$$

Here we need to know whether the inclusion on the right side of (12.6) actually holds as an equality of sets. If $\rho>0$, this equality results from a known calculation rule for the subdifferential of a sum of l.s.c. proper convex functions in finite-dimensional spaces: in fact there exists a point in the relative interior of $C=$ dom $\psi_{C}$ where $\psi_{\mathcal{A}}$ takes a finite value (see [34], Theorem 23.8). In that case, by going from (12.6) backward to (12.5), one establishes (12.4).

It is only for $\rho=0$ that the assumption of bounded friction, made once for all in the preceding, has to be used. If $\varrho=0$, the set $\mathcal{D}$ reduces to $\{0\}$, so (12.2) simply consists in the assertion:" $U$ arbitrary in $T$ and $\mathcal{R}_{T}=0$ "; one has 
to establish that such is also the meaning of (12.5) in this special situation. For $R=0$, the subdifferential $\partial \psi_{C}(R)$ consists of the polar cone $C^{\circ}$ of $C$. Because the section of $\mathcal{C}$ by the plane $\mathcal{A}$ (constructed for instance with $\rho=1$ ) is compact, $C^{\circ}$ contains the vector $-\mathcal{N}$ in its interior (see e.g. [35], parag.8.7). Consequently, the projection of $C^{\circ}$ to $I$ equals the whole of this subspace; this completes the proof.

In turn (12.4) may be transformed as follows [25]:

PROPOSITION 12.2 Define in $\mathfrak{E}_{3}$ the extended real function $\theta$

$$
\mathcal{u} \rightarrow \theta(\mathcal{u})=\frac{1}{2}\|u\|^{2}+\Psi_{\mathcal{T}}(\mathcal{u}) .
$$

Then (12.4) is equivalent to

$$
0 \in \partial \Psi_{C}(\mathcal{R})+\partial \theta(\mathcal{U})
$$

PROOF Since $\frac{1}{2}\|\|^{2}$ is a smooth function, with gradient mapping equal to identity, one has for every $\mathcal{U}$ in $\mathcal{F}_{3}$

$$
\partial \theta(\mathcal{U})=\mathcal{U}+\partial \psi_{\mathcal{T}}(\mathcal{U})
$$

Now, $\partial \Psi_{\mathcal{T}}(\mathcal{U})$ equals [ $\mathcal{N}$ ] if $\mathcal{U} \in \mathcal{T}$ and, otherwise, is empty. Therefore, $(12.8)$ is equivalent to

$$
\mathcal{U} \in \mathcal{T} \text { and } \quad 0 \in \partial \psi_{C}(\mathcal{R})+\mathcal{U}+[\mathcal{N}]
$$

which is precisely (12.4).

Incidentally, observe that resistance laws involving a pair of subdiffential mappings, as in (12.8), have a wider interest than describing contact friction in three-dimensional space. For instance, in Plasticity, this form may be used in formulating constitutive laws for which the flow rule is not 
"associated" with the yield criterion.

Recall that $\mathcal{T}$ and $\mathcal{C}$, in what precedes, depend on the position $q$ of the system, under the assumption $f(q)=0$. Let us make the same writing meaningful also for positions which do not involve contact.

To this end, we shall agree that, when $f(q)<0$, the cone $c(q)$ reduces to $\{0\}$ and that $T(q)=\mathcal{E}_{3}$. Then the relations (12.4) or (12.8) simply express that $R=0$, with $\mathcal{U}$ arbitrary in $\mathbb{R}^{n}$.

Furthermore, in what concerns evolution problems under condition $f \leqslant 0$. It is immaterial to choose any (adequately smooth) extension of the multifunction $q \rightarrow C(q) \subset \mathcal{E}_{3}$ to the case $f(q)>0$. Similarly, the linear mapping $\mathcal{G}_{q}: \mathbb{R}^{n} \rightarrow \mathcal{E}_{3}$ will be extended to such $q$, as well as the normal unit $\mathcal{K}_{q}$, with attention to preserving (3.2). The reason for such extensions lies in numerical methods, where a certain amount of violation of the desired inequality $f \leqslant 0$ has naturally to be faced.

The set of the values of $\mathcal{R} \in \mathcal{E}_{3}$ that (12.4) or (12.8) make correspond with each $U \in \mathcal{E}_{3}$ (actually the empty set if $U \in T$ ) is a cone, since the multiplication of $R$ by any strictly positive number leaves $\partial \psi_{C}(\mathcal{R})$ invariant. Like in preceding Sections, this fact will now prove essential, as we come to formulating Nonsmooth Dynamics in terms of measure differential inclusions.

In the course of a smooth motion, the contact force $\mathcal{R}$ is a function of time that we may denote by $\mathcal{P}_{\mathfrak{t}}$. This in fact is the density, relative to the Lebesgue measure $\mathrm{dt}$, of the local contact impulsion $\mathrm{d} \mathcal{P}$, an $\mathfrak{E}_{3}$-valued measure on the time interval I. For nonsmooth motions, $d \mathcal{P}$ can no more be 
expected to possess a density relative to dt, but in any case this measure may be represented in the form $\mathcal{P}_{\mathfrak{\psi}}^{\prime} \mathrm{d} \boldsymbol{u}$, where $\mathrm{d} \boldsymbol{t}$ is a nonnegative real measure on 1 and $\mathcal{P}_{\mu}^{\prime} \in \mathcal{L}^{\prime}{ }_{\text {loc }}\left(1, d \mu ; \mathcal{E}_{3}\right.$ ). The $\mathbb{R}^{n}$-valued contact impulsion $d R$, as introduced in Sect.7 for insertion into the measure equation of Nonsmooth Dynamics (7.4), has the form $d R=R_{U}^{\prime} d \mu$, and, similarly to (4.7),

$$
R_{\underline{u}}^{\prime}(t)=G_{q}^{*}(t) \mathcal{P}_{\underline{u}}^{\prime}(t)
$$

holds for every t.

As far as Coulomb's law is accepted for the description of dry friction, one naturally admits that, in possible nonsmooth motions, the density $\mathcal{P}_{\underline{u}}^{\prime}(t)$ of the local contact impulsion will be related, for every t, to the sliding velocity $u$ through the same relation as $R$ is in (12.4), or equivalently in (12.8). This, at least, raises no discussion when $t \rightarrow U$ is continuous. At instants of velocity jumps, we decide that the same relation will hold with $u$ replaced by its right-limit, namely $\mathcal{U}^{+}=G_{q} u^{+}$, since the linear mapping $\mathcal{G}_{q}: \mathbb{R}^{n} \rightarrow \mathcal{E}_{3}$ continuously depends on $q$. This assumption entails that $\mathcal{u}^{+}(t)$ belongs to $T(q(t))$ for every $t$ (an immaterial assertion when $f(q(t))<0$, since it has been agreed that $T(q(t))=\mathcal{E}_{3}$ in this event). In view of Proposition 3.1, this is equivalent to $u^{+}(t) \in T(q(t))$, a property which, in the frictionless case of Sect. 9 , has been identified as characterizing the "softness" of unilateral constraints. We thus are induced to put the following definition.

DEFINITION 12.3 The unilateral constraint investigated above is said soft with Coulomb friction if, in any motion with lo. v. velocity function. the contact impulsion has the form $d \mathcal{P}=\mathcal{P}_{\mu} d \underline{d}$, with $\mathcal{P}_{\mu}^{\prime} \in \mathcal{C}^{1}{ }_{l o c}\left(1, d \mu ; \mathcal{E}_{3}\right)$ verifying for every $\mathrm{t}$

$$
-\mathcal{U}^{+}(\mathrm{t}) \in \operatorname{proj}_{\mathcal{T}(q(\mathrm{t}))} \partial \psi_{C(q(\mathrm{t}))}\left(\mathcal{P}_{\mathcal{U}}^{\prime}(\mathrm{t})\right)
$$

(recall that $\mathcal{T}(q)=\mathcal{E}_{3}$ and $C(q)=\{0\}$ when $f(q)<0$ ) or the equivalent form 
given to a relation or this sort by Prop. 12.2

Because this relation is conical with regard to the element $\mathcal{P}_{\psi}^{\prime}(t)$, the reasoning already used in the proof of Prop. 8.2 shows that the choice of the nonnegative real measure $d y$ is immaterial, as long as dP possesses, relative to it, a density function.

REMARK 12.4 Since we have agreed to extend the definitions of $T(q)$ and $C(q)$ to positions such that $f(q)>0,(12.10)$ makes sense also in that case. But, as previously observed, this relation implies that $u^{+}(t)$ belongs to $T(q(t))$, hence to $V(q(t))$. In view of Prop 2.4 , this secures that, provided the initial data satisfy $\mathrm{f}\left(\mathrm{q}\left(\mathrm{t}_{0}\right)\right) \leqslant 0$, inequality $\mathrm{f}(\mathrm{q}(\mathrm{t})) \leqslant 0$ will hold throughout $\mathrm{l}$.

REMARK 12.5 The validity of $(12.10)$ for $t=t_{0}$ calls for some comments. In beginning this section, we recalled the meaning given to the initial condition $u\left(t_{0}\right)=u_{0}$ of an evolution problem. It is understood that $u_{0}$ equals the left-limit $u^{-}\left(t_{0}\right)$ in some anterior motion, during which the unilateral constraint was already in effect. In particular, one may have $f\left(q_{0}\right)=0$ and $u_{0}$ interior to the half-space $-V\left(q_{0}\right)$; this implies that a collision takes place at instant $t_{0}$. Then sof tness, as expressed by $(12.10)$, makes that $u^{+}\left(t_{0}\right)$ belongs to the linear space $T\left(q_{0}\right)=V\left(q_{0}\right) \cap-V\left(q_{0}\right)$. But the case $f\left(q_{0}\right)=0$ with $u_{0}$ interior to $V\left(q_{0}\right)$, i.e. initial velocity implying contact break, is excluded from the present study. This will cause no great inconvenience in practice.

REMARK 12.6 Put the notation $\mathcal{G}_{q}(V(q))=\mathcal{V}(q)$; this is the closed half-space of $\mathcal{E}_{3}$ lying on the same side as $\mathcal{K}_{q}$ with respect to $\mathcal{T}(q)$. The set $\partial \psi_{C}\left(\mathcal{P}_{p}^{\prime}\right)$ is contained in $C^{\circ}$, the polar cone of $C$, which in turn is contained in the halfspace $-\mathcal{V}(q)$. Then, in $(12.10)$, the operation $\operatorname{proj}_{\mathcal{T}(q)}$ might equivalently be 
replaced by the proximation mapping to $V(q)$. This would be numerically inconvenient, but is llable to improve the consistency of some further developments.

REMARK 12.7 In applications, describing dry friction through Coulomb's law can only provide a rather crude approximation. However, as this law retains the essential features of the phenomenon, its use at the stage of a first study is extremely valuable in numerous situations. As soon as this law has been adopted, under its traditional form, there is little doubt that its generalization (12.10) can also be accepted for every motion in which the velocity function is continuous, even in the absence of local absolute continuity. We shall discuss later its use in the case of a velocity jump.

\section{TWO-DIMENSIONAL CONTACT}

In the same setting as in the above Section, we now make the following additional assumption. However three-dimensional the contact may physically be, we shall suppose that, for every $q$ such that $f(q)=0$ (and also for $f(q) \geqslant 0$, after the extension we have agreed to make) the range $G_{q}\left(\mathbb{R}^{n}\right)$ or $G_{q}$ reduces to a two-dimensional subspace $\mathcal{W}_{\mathrm{q}}$ of $\mathcal{E}_{3}$. Such is the case, for instance, if the part $\mathcal{B}_{1}$ of the system is astrained, by the primitive constraints, to only perform motions parallel to a fixed plane. The condition

found in Remark 3.2, in order that $G_{q}^{*} \mathcal{N}_{q} \neq 0$ will be supposed fullfilled, i.e. $W_{\mathrm{q}}$ and the tangent plane $\tau_{\mathrm{q}}$ are distinct : let us denote by $I$ a unit vector of their intersection.

Due to the expression (4.7) of the covariant components of the contact force $\mathcal{R}$ (or, in Nonsmooth Dynamics, the covariant components of the density $\mathcal{P}_{\hat{U}}(t)$ of contact impulsion), the dynamically significant information 
concerning this vector is entirely conveyed through its equivalence class modulo ker $\mathcal{G}_{\mathrm{q}}^{*}$. This kernel equals the subspace of $\mathcal{E}_{3}$ orthogonal to $w_{q}$, consequently, we may in the sequel replace $\mathcal{R}$ by its orthogonal projection to $w_{q}$, also called $R$, by abuse of notation.

We shall come back, in Sec.17, to a discussion of what in general becomes the three-dimensional law of friction under such a geometric two-dimensional reduction. Let us restrict ourselves at present to the usual case where the result is simply the familiar two-dimensional version of Coulomb's law. The considerations of the preceding section might readily be adapted to this case. Here we shall rather choose to express the twodimensional Coulomb law in the following alternative form. For more readibility, the subscript q will momentarily be omitted.

There exist in the vector plane $W$ two half-lines $\mathcal{D}_{+}$and $\mathcal{D}_{-}$, emanating from the origin and lying, with respect to $T$, on the same side as $\mathcal{N}$. The convex cone $C$ now equals their convex hull. In smooth motions, the sliding velocity is essentially an element of $T \cap W$, say $U=s ?$ with $s \in \mathbb{R}$. coulomb's law consists or the three implications

$$
\begin{aligned}
& s>0 \Rightarrow R \in \mathcal{D}_{+} \\
& s<0 \Rightarrow R \in \mathcal{D}_{-} \\
& s=0 \Rightarrow R \in C
\end{aligned}
$$

The angles that $\mathcal{D}_{+}$and $\mathcal{D}_{-}$make with I have respective tangent equal to $-1 / \gamma_{+}$and $1 / \gamma_{-}$, where the positive real numbers $\gamma_{+}$and $\gamma_{-}$respectively are the familiar friction coefficients corresponding to positive and negative sliding.

All these elements are defined for such $q$ that $f(q)=0$; as before, we shall imagine an extension of them to every $q$ such that $f(q) \geqslant 0$ (at least in 
a neighborhood of hypersurface $f=0$ ).

The vector plane $w$ depends on $a$; it will prove conventent to send it onto a fixed copy of $\mathbb{R}^{2}$, called the calculation plane, equipped with the usual base consisting of vectors $\mathbf{i}=(1,0)$ and $\mathbf{j}=(0,1)$. To this end, $a$ regular linear mapping $\mathcal{F}_{q}: w_{q} \rightarrow \mathbb{R}^{2}$ will be constructed, in such a way that $\mathcal{F}_{q} I$ is a positive vector of the first axis. Denote by $G_{q}: \mathbb{R}^{n} \rightarrow \mathbb{R}^{2}$ the product mapping $\mathcal{F}_{q} G_{q}$. Assume that the real function $f$ is at least $C^{2}$; then it will be possible to choose $\mathcal{F}_{q}$ in order that $G_{q}$ depend on $q$ in a $C^{\prime}$ way.

Let us mean by $\mathcal{F}_{q}^{*}$ the transpose of $\mathcal{F}_{q}$, in the sense of the natural scalar products of $\mathbb{R}^{2}$ and $W$, and denote by $D_{+}$and $D_{-}$the images of $\mathcal{D}_{+}$and $\mathcal{D}_{\text {_ under }} \mathcal{F}_{\mathrm{q}}^{*-1}$.

There would remain enough arbitrariness in the choice of $\mathcal{F}_{q}$ for making these images equal two fixed half-lines of $\mathbb{R}^{2}$, e.g. the half-lines generated by $(-1,1)$ and $(1,1)$. The drawback is that the two friction coefficients $f_{+}$ and $f_{\text {_ }}$ would then have to depend smoothly on $q$. So we shall not use this trick in the sequel and only assume that the convex hull, say $\mathrm{C}$, of $\mathrm{D}_{+}$and D_ contains $\mathrm{J}$ in its interior.

Under these notations, the friction law (13.1) to (13.3) may equivalently be formulated as a relation of the same form between $R=\mathcal{F}_{q}^{*-1} \mathcal{R}$ and $U=$ $\mathcal{F}_{q} U=G_{q} U=\sigma i$. Due to the assumptions made, $\sigma$ is a real number of the same sign as $s$ or vanishing with it. Henceforth, there only is to replace $s, \mathcal{D}_{+}$, $\mathcal{D}_{-}, C$ respectively by $\sigma, D_{+}, D_{-}, C$.

In order to express the dynamics of the considered system, one has to bring together the above reaction law and the Lagrange equations. The covariant components of the contact force make the element $r$ of $\mathbb{R}^{n}$, related to $R$ through 


$$
r=G_{q}^{*} R=G_{q}^{*} \mathcal{F}_{q}^{*} R=G_{q}^{*} R
$$

After having so restated the two-dimensional law of Coulomb, we now propose to extend it to Nonsmooth Dynamics, in the same line as in Sec. 12. Recall that, in such a context, velocity functions are significant only through their one-sided limits. Instead of $u, U$ and $U$, the right-limits $u^{+}, U^{+}=G_{q} u^{+}$ and $\mathrm{U}^{+}=\mathrm{G}_{\mathrm{Q}} \mathrm{U}^{+}$are introduced into the above writing. The contact force $R$ is replaced, for every $t$, by the value $\mathcal{P}_{\underline{U}}^{\prime}(t)$ of the density function of the three-dimensional contact impulsion, or equivalently by the orthogonal projection of $\mathcal{P}_{\nu}^{\prime}(\mathrm{t})$ to $\mathcal{W}(q(\mathrm{t}))$. When coming to the use of the calculation plane, the function $P_{\mu}^{\prime}=\mathcal{F}_{q}^{*-1} \mathcal{P}_{\mu}^{\prime}$ is considered.

The dynamics of the system is now expressed by the Lagrange equation in $\mathbb{R}^{n}$

to be joined with

$$
A(q) u_{u}^{\prime}-k(t, q, u) t_{u}^{\prime}=R_{u}^{\prime}
$$

$$
\begin{aligned}
& R_{U}^{\prime}=G_{Q}^{*} P_{U}^{\prime} \\
& \exists \sigma \in \mathbb{R}: \quad U^{+}=\sigma i \\
& \sigma>0 \Rightarrow P_{\mu}^{\prime} \in D_{+} \\
& \sigma<0 \Rightarrow P_{U}^{\prime} \in D_{-} \\
& \sigma=0 \Rightarrow P_{\mu}^{\prime} \in C,
\end{aligned}
$$

for every t such that $f(q(t)) \geqslant 0$.

on the contrary, when $f(q(t))<0$, then $R_{u}^{\prime}=0$.

\section{VELOCITY JUMPS IN FRICTIONAL DYNAMICS}

The formulation given above, for the Nonsmooth Dynamics of a system with single possible contact and two-dimensional coulomb friction, will 
now be used in discussing the event of a velocity jump.

This means that, at some instant $t_{s}$, the $\mathbb{R}^{n}$-valued measure du possesses an atom, the mass of which equals

$$
u^{+}\left(t_{s}\right)-u^{-}\left(t_{s}\right)=u_{u}^{\prime}\left(t_{s}\right) u_{s} \text {. }
$$

Here, the positive number $\mu_{s}$ is the mass of the atom that the measure $d y$ should possess at point $t_{s}$.

Recalling that the Lebesgue measure dt has no atom, one derives from (13.5) that, at $t=t_{s}$.

$$
u^{+}-u^{-}=A_{Q}^{-1} G_{q}^{*} P_{u}^{\prime} \mu_{s}
$$

If $f\left(q\left(t_{s}\right)\right)<0$, i.e. no contact, one has $P_{u}^{\prime}\left(t_{s}\right)=0$, so no jump of $u$ can occur. We therefore shall assume $f\left(q\left(t_{s}\right)\right) \geqslant 0$ (equivalently $f\left(q\left(t_{s}\right)\right)=0$ ) and denote simply by $G$ the value of $G_{q}$ at $q=q\left(t_{s}\right)$. Then, for $t=t_{s}$, one has $R_{u}^{\prime}=G^{*} P_{u}^{\prime}$. Put $P_{\mu}^{\prime}\left(t_{s}\right) \mu_{s}=P$ and apply the linear mapping $G: \mathbb{R}^{n} \rightarrow \mathbb{R}^{2}$ to both members of (14.1); this yields

$$
\mathrm{U}^{+}-\mathrm{U}^{-}=H P,
$$

where $H$ denotes the symmetric $2 \times 2$ positive definite matrix $G A^{-1} G^{*}$.

This has to be joined with two-dimensional Coulomb law, expressed as in Sec. 13 by

$$
\begin{aligned}
\exists \sigma \in \mathbb{R}: \quad U^{+}=\sigma 1 \\
\sigma>0 \Rightarrow P \in D_{+} \\
\sigma<0 \Rightarrow P \in D_{-} \\
\sigma=0 \Rightarrow P \in C^{-}
\end{aligned}
$$

We are to discuss how, starting with given $u^{-}$, the system of conditions (14.1) to (14.6) allows one to determine $u^{+}$.

Combining (14.2) and (14.3), one obtains

$$
P=-H^{-1} U^{-}+O H^{-1} \mathbf{i}
$$


which expresses that $P$ belongs to the line $\Delta$ of $\mathbb{R}^{2}$ drawn through the point $-H^{-1} U^{-}$, with $H^{-1}$ as directing vector. Easy calculation (for instance by observing that $\Delta$ is orthogonal to $H \mathbf{j}$ ) yields that this line intersects the second axis at point

$$
S=-\left(\mathbf{j} \cdot U^{-}\right) \mathbf{j} /(\mathbf{j} \cdot \mathbf{H} \mathbf{j}) \text {. }
$$

Due to the way we have chosen the mapping $\mathcal{F}$, the real number $\mathbf{j} \cdot U^{-}=\mathcal{F}^{*} \mathbf{j} \cdot \mathcal{U}^{-}$has the same sign as $\mathcal{N} \cdot \mathcal{U}^{-}=\mathcal{G}_{q}^{*} \mathcal{N} \cdot U^{-}$or vanishes with it. Through Prop.3.1, one concludes that the coordinate of $S$ on the $j$ axis is positive, negative or zero if and only if the same is true for $u^{-} . \nabla f\left(q\left(t_{s}\right)\right)$.

First case: impact.

This is the event where $f\left(q\left(t_{s}\right)\right)=0$, with $u^{-}\left(t_{s}\right), \nabla f\left(q\left(t_{s}\right)\right)>0$. Consequently $f(q(t))<0$ on some left-neighborood of $t_{s}$, i.e. $t_{s}$ is an instant of nontangential collision. The point $S$ lies in the interior of $C$, so the line $\Delta$ intersects $C$ and does not pass through the origin. Observe that $\mathbf{i} . H^{-1} \mathbf{i}>0$, i.e. the vector $H^{-1} \mathbf{i}$ directs $\triangle$ from left to right. Therefore, the point $P$, as expressed in (14.7), lles on the right of $\mathrm{H}^{-1} U^{-}$if $\sigma>0$ and on the left in the reverse case. By comparison with (14.4) to (14.6), one concludes that the formulated set of conditions is satisfied if and only if $P$ equals the nearest point to $\mathrm{H}^{-1} \mathrm{U}^{-}$in the intersection $\triangle \cap \mathrm{C}$.

So the problem of determining $\mathrm{u}^{+}$possesses a unique solution in that case.

Second case : sliding.

We now assume that $f\left(q\left(t_{s}\right)\right)=0$, with $u^{-}\left(t_{s}\right), \nabla f\left(q\left(t_{s}\right)\right)=0$ (equivalently $u^{-}\left(t_{s}\right) . \nabla f\left(q\left(t_{s}\right)\right) \leqslant 0$, since the anterior motion is assumed to agree with $\left.f \leqslant 0\right)$. 
Then $S=0$, i.e. the line $\triangle$ passes through the origin.

- If $\Delta \cap C$ consists only of the origin, the formulated set of condition admits $\mathrm{P}=0$, nence $\mathrm{u}^{+}=\mathrm{U}^{-}$, as unique solution: no velocity jump occurs.

- If $\Delta$ intersects also the interior of $C$, one has to determine whether it is possible for $P$ to lie in this interior. In view of conditions (14.4) to (14.6), this requires $\mathrm{U}^{+}=0$. It is in fact a solution if $-\mathrm{H}^{-1} \mathrm{U}^{-}$eint $\mathrm{C}$ : then the sliding suddenly sticks. Concurrently $\mathrm{P}=0$, with $\mathrm{u}^{+}=\mathrm{u}^{-}$, i.e. no velocity jump, is also a solution in this case.

- If $\Delta$ contains one of the half-lines $D_{+}$or $D_{-}$, a value of $P$ on this half-line meets the requirements provided the resulting value of $\mathrm{U}^{+}=$ $\mathrm{U}^{-}+\mathrm{HP}=\sigma \mathrm{i}$ agrees with conditions (14.4) to (14.6). For instance, imagine $D^{+} \subset \Delta$ : these conditions require $\sigma \geqslant 0$. This can happen only if $-H^{-1} U^{-} \in D_{+}$and then every value of $\mathrm{P}$ belonging to the line segment $\left[-\mathrm{H}^{-1} \mathrm{U}^{-}, 0\right]$ is a solution. The corresponding values of $\mathrm{U}^{+}$cover the line segment $\left[0, \mathrm{U}^{-}\right]$.

REMARK 14.1 The latter is the most interesting item of the discussion. It shows that, contrary to the frictionless case, an episode of smooth motion with persistent contact may end with a velocity jump without any collision occurring. This is a dynamical analogue to the locking effect, well known in the statics of mechanisms with dry friction.

The earliest reference we know of, where such a possibility is asserted, is a note by L. Lecornu [26]. At the time, a controversy has been opened by $P$. Painleve [36], with the observation that, in systems involving Coulomb friction, some initial value problems could have no solution, or also several solutions. In addition, the behaviour of the system depended on its constants in a discontinuous way. To Painlevé, and later to E. Delassus [37][38], these findings seemed in contradiction with the very bases of Physics. In the 
subsequent years, different opinions were sustained by such authors as $F$. Klein [39], R.v.Mises [40], G. Hamel [41] or L. Prandtl [42]. Even after $H$. Beghin [43][44] had clearly demonstrated that the incriminated findings actually agreed with common observation, some suspicion remained in the scientific community that Coulomb's law could be intrinsically illogical.

Today, one is accustomed to meet multiple solutions or the absence of solution to physical problems, usually ascribing these facts to the nature of the treated information, without opening any discussion about determinism in Physics. One is also familiar with discontinuous behaviour.

Dynamic locking, that we propose to call a frictional catastrophe, is commonly observed in practice. The example of the chattering motion of a piece of chalk driven at an angle against a blackboard, so that a dotted line is drawn, was already put forward by E. Delassus [37]. A model of this phenomenon is presented in [30], with some drawings generated by a computer using the time discretization procedure of Sect. 15 below. This displays an instance of a "stick-slip" motion. Depending on the system constants, frictional catastrophes and intermittent contact breaking may occur or not. No attempt so far has been made at comparing this model, quantitatively, with experimental measurements.

A very simple example of frictional catastrophe is presented in Sect.15, as a demonstration of the ability of our numerical technique to handle nonsmooth solutions.

REMARK 14.2 From the mathematical standpoint, we think that little has to be retained of the early discussions on the subject. At the time, differential equations were implicitely understood in the sense of the elementary theory: 
solutions should possess some regulated functions. as their derivatives of the highest order involved. In other words, it was admitted that the acceleration $q^{\prime \prime}$ possessed a right-limit and a left-limit at every t. Certain of these limits played an essential role in the discussions by Painleve and his followers unfortunately, in usual instances where an interval of smooth motion precedes the catastrophic instant $t_{s}$, one finds that the norm of $q$ " actually tends to inf inity when $t$ tends to $t_{s}$ from the left. The concept of a measure differential inclusion, on which the present lectures are based, provides a more synthetic view, since it allows one to express Dynamics on the whole interval 1 , including $t_{s}$, and does not rely on the existence of one-side accelerations.

REMARK 143 There remains to discuss whether the velocity jumps agreeing with the constraint law (12.10) are physically realistic. We have already stressed that, even in the absence of friction, predicting safely the outcome of a shock would require some high order of information, actually unavailable in engineering situations. Things naturally become worse if friction is entered into account. What do we know about the Physics of high pressure friction, during the "very short" interval of time on which the velocity change takes place? Already for the frictionless case, we have in Sect. 6 been reluctant in accepting the invariance of the direction of $\nabla f(q)$ during this interval. The latter invariance, if admitted, makes of the no-friction assumption a time-independent linear condition imposed on the contact force at every instant; therefrom, the normality of the contact percussion is inferred, by integration on the interval. On the contrary, Coulomb law imposes on contact force a nonlinear condition. Even if one assumes this condition independent of time, it cannot be expected in general to commute 
with integration. The contact percussion can safely be asserted to verify (12.10) only as far as the sliding velocity is sure to remain zero or to keep a constant direction for the short duration of the investigated shock. It is of course in the case of two-dimensional friction that the latter event proves the easiest to discuss.

The reader may find in [2] an attempt at analysing frictional impact, in the line formerly suggested by $G$. Darboux. This consists in investigating the variation of the velocity as a function of some "micro-time", relatively to which the system position remains a constant. Even so, the conclusion is subject to some assumption about the shock end which seems difficult to justify.

In conclusion, the concept of a frictional and soft contact, as involved in Definition 12.3, only generates a special model of frictional shock, with the advantage of good theoretical consistency. As we shall see in the next Section, the corresponding motions are also very tractable numerically. Physical situations to which this model is relevant very probably exist, but experimentation is still needed to safely identify them in practice.

\section{ALGORITHMFOR TWO-DIMENSIONAL FRICTION}

We now present a time-discretization procedure for computing a motion under the conditions of Sec.13. The notations are the same as in the frictionless case, exposed in Sec. 11.

Stage 1. Calculate $t_{M}=t_{1}+\frac{1}{2} h$, the midpoint approximants

$$
q_{M}=q_{1}+\frac{1}{2} n u_{1} \in \mathbb{R}^{n}, \quad A_{M}=A\left(q_{M}\right) \in \mathbb{R}^{n \times n}, \quad K_{M}=K\left(t_{M}, q_{M}, u_{1}\right) \in \mathbb{R}^{n}
$$

and the "loose velocity"

$$
u_{L}=u_{1}+n A_{M}^{-1} K_{M}
$$


As in Sec.13, a single constraint inequality, say $f \leqslant 0$, is taken into account. If $f\left(q_{M}\right)<0$ or $u_{L} . \nabla f\left(q_{M}\right) \leqslant 0$, then make $u_{F}=u_{L}$ and go to stage 3 .

Stage 2. If, on the contrary, $f\left(q_{M}\right) \geqslant 0$ and $u_{L} \cdot \nabla f\left(q_{M}>0\right.$, contact is estimated to have effect on the considered time-step. One constructs a discrete analog to the measure differential inclusion of Dynamics

$$
d u=A^{-1}(q) K(t, q, u) d t+A^{-1}(q) d R
$$

by equalling some approximate values of the integrals on $\left(t_{1}, t_{F}\right)$ of the respective members, namely

$$
u_{F}-u_{1}=n A_{M}^{-1} K_{M}+A_{M}^{-1} R \text {. }
$$

Let $G_{M}$ denote the value taken at $q=a_{M}$ by the linear mapping $G_{q}: \mathbb{R}^{n} \rightarrow \mathbb{R}^{2}$ defined in Sec. 13. Then an approximate version of (13.7) reads

$$
R=G_{M}^{*} P \text {, }
$$

where $P$, an element of the calculation plane, is an approximant of the total contact impulsion on the interval $\left(t_{1}, t_{F}\right)$. Putting $U_{1}=G_{M} U_{1}$ and $U_{F}=G_{M} U_{F}$, one derives from (15.1) that

$$
U_{F}-U_{1}=h G_{M} A_{M}^{-1} K_{M}+H_{M} P
$$

Here, similarly to what has been done in Sec.13, one denotes by $H_{M}$ the symmetric positive def inite $2 \times 2$ matrix $G_{M} A_{M}^{-1} G_{M}^{*}$.

According to the decision made in Sec.11, of considering $U_{F}$ as a simulation of $\mathrm{U}^{+}$, the softness condition (13.7) will, in the present discretization procedure, be transcribed as

$$
\exists \sigma \in \mathbb{R}: \quad U_{F}=\sigma \mathbf{i} .
$$

Discretizing the two-dimensional Coulomb law consists in relating $P$ to $U_{F}$ by the system of implications

$$
\begin{aligned}
& \sigma>0 \quad \Rightarrow \quad P \in D_{+} \\
& \sigma<0 \quad \Rightarrow \quad P \in D_{-}
\end{aligned}
$$




$$
\sigma=0 \quad \Rightarrow \quad P \in C \text {. }
$$

In the case where the friction coefficients depend on $q$, the elements $D_{+}, D_{-}$ and $C$ will be evaluated at $u=a_{M}$.

The determination of $P$ and $u_{F}$ from this set of conditions is similar to what has been done in one of the cases of shock investigated in Sec. 14. If one puts $U_{L}=G_{M} U_{L},(15.2)$ becomes

$$
U_{F}=U_{L}+H_{M} P \text {. }
$$

By combination with (15.3), this yields

$$
\exists \sigma \in \mathbb{R}: \quad P=-H_{M}^{-1} U_{L}+\sigma H_{M}^{-1} \mathbf{i} .
$$

This expresses that $P$ belongs to the line $\Delta$ of $\mathbb{R}^{2}$, drawn through the known point $-H_{M}^{-1} U_{L}$, with known directing vector $H_{M}^{-1} \mathfrak{i}$. This line intersects the second axis of $\mathbb{R}^{2}$ at point

$$
S_{M}=-\left(\mathbf{j} \cdot U_{L}\right) \mathbf{j} /\left(\mathbf{j} \cdot H_{M} \mathbf{j}\right)
$$

A similar expression was discussed in (14.8). Since the present computation stage is developed under the assumption $u_{L} . \nabla f\left(q_{M}\right)>0$, one finds in the same way as in Sec.14 that $\mathrm{j} . U_{L}<0$, so $S_{M}$ is sure to be interior to $C$. Observe in addition that $P$ lies in $\triangle$ on the right or on the left of $-H_{M}^{-1} U_{L}$ according to the sign of $\sigma$. One concludes to the existence of a unique solution $P$, characterized as the nearest point to $-H_{M}^{-1} U_{L}$ in the intersection $\Delta \cap C$.

For computation it is more convenient to formulate the same as follows:

- If $-H_{M}^{-1} U_{L} \in C$, then $P=-H_{M}^{-1} U_{L}$.

$$
\text { - Otherwise } \begin{aligned}
-H_{M}^{-1} U_{L} \cdot \mathbf{i}<0 \Rightarrow P=\Delta \cap D_{+} \\
-H_{M}^{-1} U_{L} \cdot \mathbf{i}>0 \Rightarrow P=\Delta \cap D_{-} .
\end{aligned}
$$

After $P$ is calculated, one derives $u_{F}$ from (15.1), namely

$$
u_{F}=u_{L}+A_{M}^{-1} G_{M}^{*} P
$$


Stage 3 . The computation step finishes with $q_{F}=q_{M}+\frac{1}{2} h u_{F}$.

REMARK 15.1 A more intuitive description of the above discretization procedure may be found in [24]. Instead of relying on the calculation plane, it uses the image of $C$ under $\mathcal{G}_{q}^{*-1}$, a two-dimensional cone in $\mathbb{R}^{n}$. This makes the comparison with the frictionless case clearer, but numerically is less effective. The calculation plane is also useful at the stage of deriving the inequalities needed in the study of existence and regularity of solutions.

REMARK 15.2 The case $-H_{M}^{-1} U_{L} \in C$ yields $U_{F}=0$, i.e. zero sliding velocity (the discussion here is simpler than that of the similar geometric construction made in Sec. 14, because $S_{M}$ is certainly interior to $\mathrm{C}$.

In that connection, the algorithm works very well to compute a motion involving the event which, in Remark 14.1, we have called a frictional catastrophe. Now, we have just seen that each computation step is deterministic, i.e. it yields a unique pair $a_{F}, u_{F}$. This contrasts with the conclusions of Sec.14, showing multiple possible outcomes for such a catastrophe: all the points of a line segment are solutions in what concerns the contact percussion $P$ and similarly in what concerns $U^{+}\left(t_{s}\right)$ or $U^{+}\left(t_{s}\right)$ (the latter may take any value between zero and $\left.\mathrm{U}^{-}\left(\mathrm{t}_{\mathrm{s}}\right)\right)$.

In fact, the algorithm is able to approximate any of these solutions. As soon as the successive discretization intervals are chosen, a sequence of values of $u_{F}$ is unambiguously generated. This sequence is smooth, except for a jump in one of the intervals, said catastrophic. Before this jump, the computation of the motion, from given initial data $q\left(t_{0}\right), u\left(t_{0}\right)$, yields consistent results, for arbitrarily fine discretizations. But the value of the jump obtained in a catastrophic interval depends on the ratio in which the 
exact instant divides this interval. So, calculations made with different discretization meshes may yield different results af ter the catastrophe.

EXAMPLE 15.3 A round-tipped rigid body $\mathcal{B}_{1}$ performs motions parallel to a vertical plane. It is submitted to gravity and confined by a horizontal fixed boundary $\mathcal{B}_{0}$, with friction coefficient equal to 0.5 . Initial conditions are those of contact, with negative angular velocity and sliding velocity directed to the right.

On Figure 15.1, the computer has drawn the profile of $\mathcal{B}_{1}$ for every third step of the time-discretization (numbers refer to these steps). After an epi-

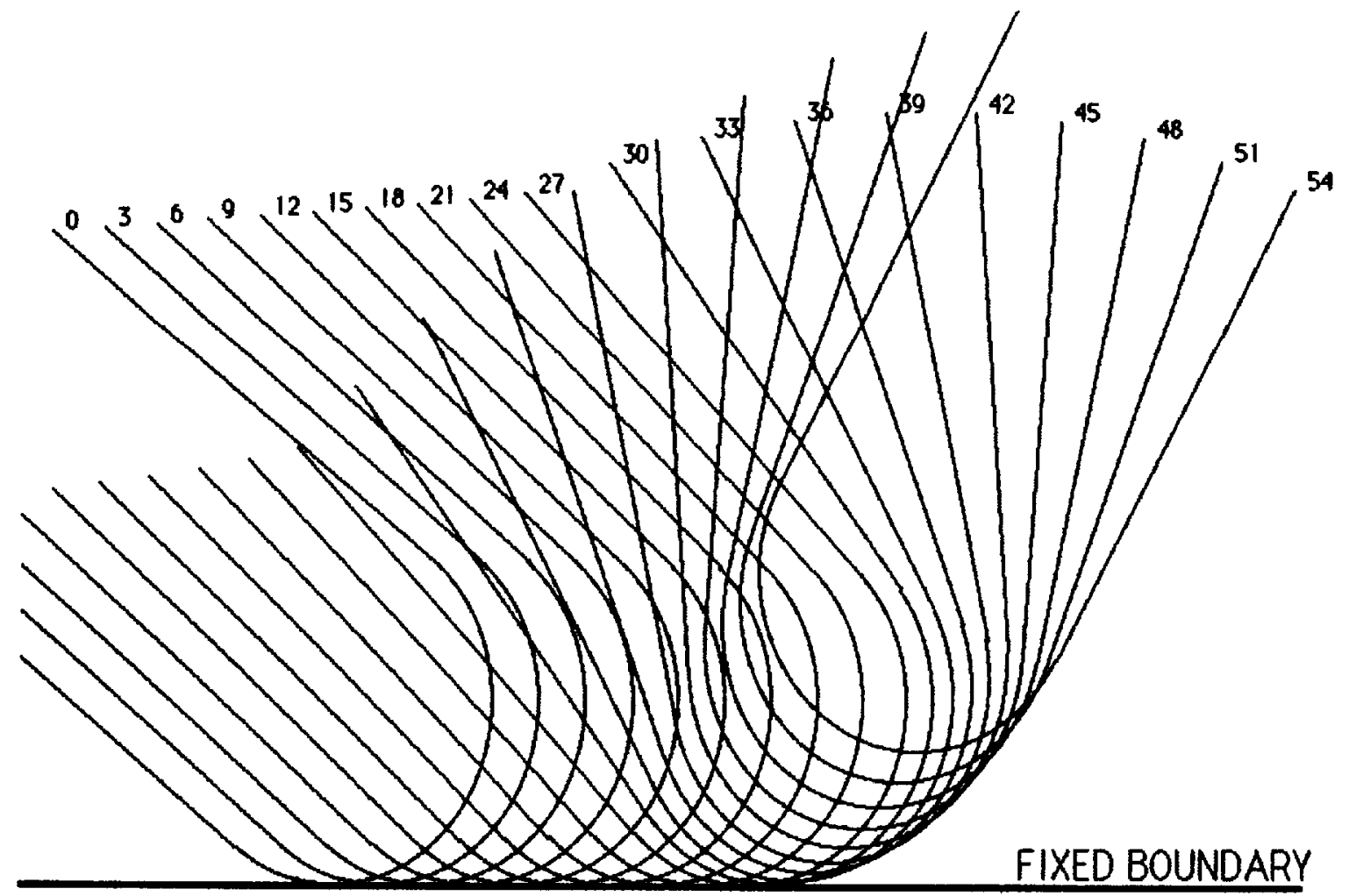

Figure 15.1

sode of persistent contact with sliding of constant direction, a catastrophe occurs. The horizontal component of the velocity of the lowest particle of $\mathfrak{B}_{1}$ (this equals the sliding velocity in the case of contact) presents a sudden 
drop. At the same instant, contact is broken, with zero normal velocity. In the process, a percussion is imparted to $\mathcal{B}_{1}$ from $\mathcal{B}_{0}$. making the negative angular velocity increase in magnitude.

Though discretization is rather rough, the corrective procedure of the possible constraint violation, described in Remark 11.1 , has not been used. Drawing is however found to comply very well with the unilateral constraint.

On Figure 15.2, the horizontal component of the velocity of the lowest particle is plotted versus time. All curves correspond to the same initial conditions as above, but are computed with finer discretization. In order to display the multiple possible outcomes of the catastrophe, computation has

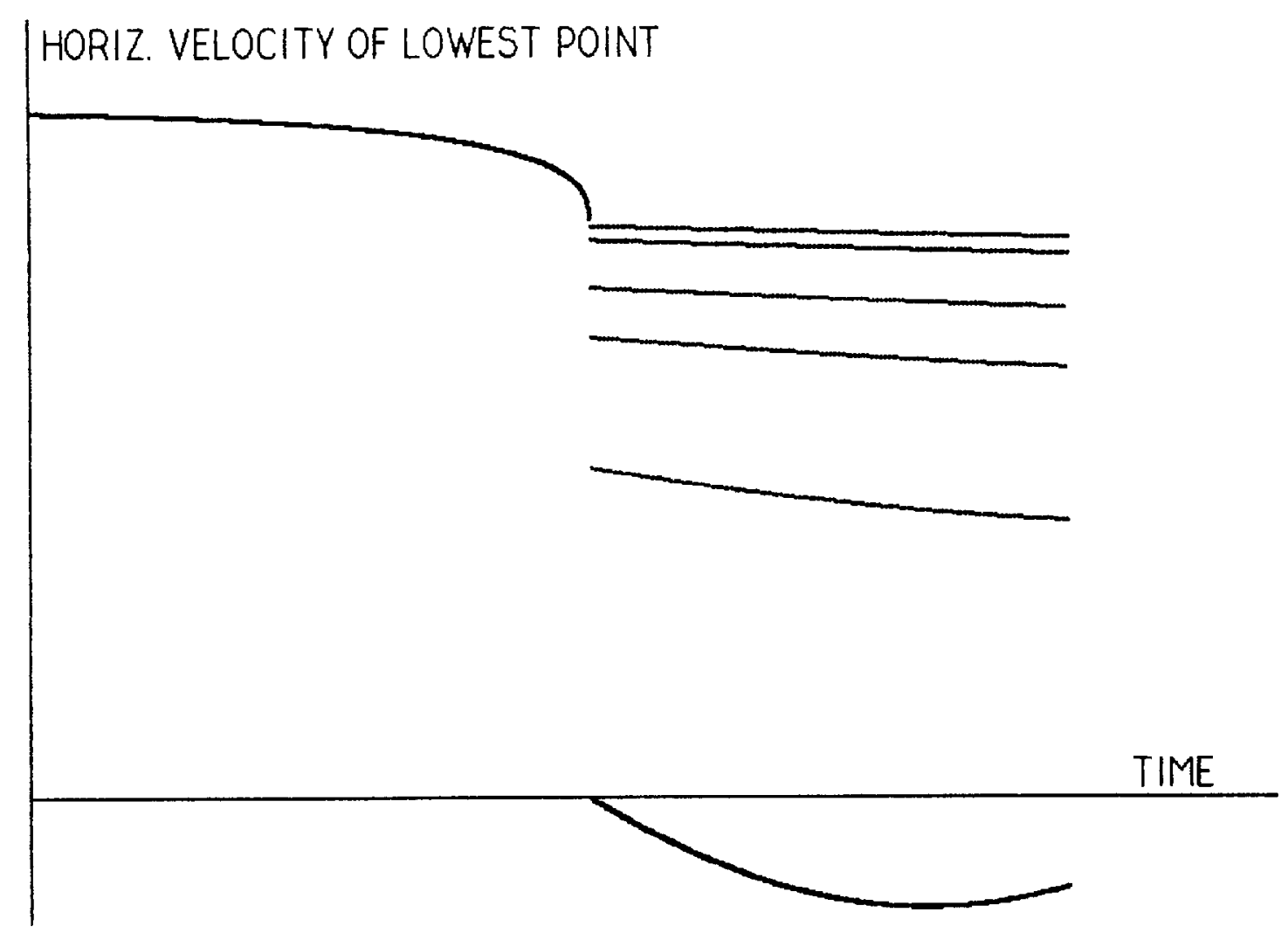

Figure 15.2

been repeated, each time with uniform time-mesh, but successively using different values of the step-length, namely 


$$
h=0.00046-0.0001 * \text { RND. }
$$

Here RND denotes the built-in random sequence of the computer, with values in $[0,1[$. The maximal catastrophe, i.e. sliding velocity dropping to zero, is repeatedly obtained, more than two times out of three on an average, and followed with a well defined contact-free motion. The other curves show various sliding velocity drops of smaller amplitudes. More extensive experimentation has confirmed that each value of the sliding velocity drop corresponds to a well defined consequent motion. Statistically, the values of the drop are not uniformly distributed: frequency is found maximal in the vicinity of zero drop.

As expected, consistent results are obtained regarding the motion prior to the catastrophe. It is apparent that the rate of change of the sliding velocity tends to $-\infty$ on the left of the catastrophic instant. The acceleration $q^{\prime \prime}: l \rightarrow \mathbb{R}^{n}$ thus beeing unbounded, cannot possess a left-limit at the catastrophic instant.

\section{AN EXAMPLE OF THREE-DIMENSIONAL FRICTION}

We suppose in this section that the system consists of a single particle of unit mass, confined in a region $\Phi$ of physical space by an unmoving boundary $\Sigma$. This is a model of a small object which, in the case of contact with the boundary, is assumed to slide on it, without rolling nor tumbling. Then $q^{1}, q^{2}, q^{3}$ may be identified with the particle coordinates, relative to some inertial orthonormal Cartesian frame and $A(q)$ consists, for every $q$, in the unit matrix.

In addition to the possible frictional reaction of the boundary, the 
particle is submitted to a force given as a smooth function $F$ of $t, q, u$. The case of a boundary with prescribed motion may be reduced to this one, through changing the reference frame; there only is to include in $F$ the fictitious forces, thanks to which the new reference frame may be treated as inertial.

The particle dynamics is expressed by this equality of $\mathcal{E}_{3}$-valued measures on the time-interval I

$$
d u=d R+F(t, q, u) d t,
$$

i.e. after representing vector measures by density functions relative to some nonnegative real measure $d \mu$,

$$
u_{u}^{\prime}(t)=R_{u}^{\prime}(t)+F(t, q, u) t_{u}^{\prime}(t)
$$

an equality to be satisfied for every $t \in l$.

In the present case, the mapping $G_{q}$ reduces to identity for every $q$. Then, using the law of frictional contact in the form (12.8), one obtains the measure differential inclusion

$$
0 \in \partial \Psi_{C(q)}\left(u_{u}^{\prime}-F(t, q, u) t_{u}^{\prime}\right)+\partial \theta_{q}\left(u^{+}\right) \text {. }
$$

The feasible region $\Phi$ of $\mathbb{R}^{3}$ is defined as before by a single inequality $f(q) \leqslant 0$. For $f(q)=0, C(q)$ denotes the friction cone at the point $q$ of the boundary. Again, let us agree to extend its definition, in a smooth arbitrary way, to the values of $q$ such that $f(q) \geqslant 0$. In addition, $C(q)$ is interpreted as reducing to the zero of $\mathbb{R}^{3}$ when $f(q)<0$. With every a such that $f(q) \geqslant 0$, the vector plane $T(q)$ also is associated, orthogonal to $\nabla f(q)$. For $f(q)<0$, we agree to understand $T(q)$ as consisting of the whole of $\mathbb{R}^{3}$. The extended-real function $\theta_{q}$, as defined in (12.7), equals $\frac{1}{2}\|.\|^{2}+\psi_{T(q)}$.

In particular, at every $t$ such that $f(q(t))<0$, one has $\partial \theta_{q(t)}(x)=\{x\}$ for every $x \in \mathbb{R}^{3}$, while the value of $\partial \psi_{C(q(t))}(x)$ equals $\mathbb{R}^{3}$ for $x=0$ and $\varnothing$ 
otherwise. Therefore (16.2) reduces in that case to the differential equation $u_{t}^{\prime}-F(t, q, u)=0$ of contact-free motion.

Recall that a condition such as (16.2) implies that $q(t) \in \Phi$ for every $t$, as soon as this is assumed to hold for $t=t_{0}$.

Some cases of existence, for the solutions to the coresponding initial value problem, are investigated in [13].

Here we shall only present a time-discretization method for their approximate computation [25]. With the same notations as in previous sections, each time-step runs as follows.

Stage 1. Calculate $t_{M}=t_{1}+\frac{1}{2} h$, the midpoint approximant $q_{M}=q_{1}+\frac{1}{2} h u_{1}$, the force estimation $F_{M}=F\left(t_{M}, q_{M}, u_{1}\right)$ and the "loose velocity" $u_{L}=u_{1}+n F_{M}$.

\section{Stage 2.}

- If $f\left(q_{M}\right)<0$ or $u_{L} . \nabla f\left(q_{M}\right) \leqslant 0$, then $u_{F}=u_{L}$

- Otherwise, $u_{F}$ is determined by a semi-implicit discretization of (16.2). In view of the positive homogeneity of the multifunction $\partial \Psi_{C}$, this is

$$
0 \in \partial \Psi_{C}\left(u_{F}-u_{L}\right)+\partial \theta\left(u_{F}\right)
$$

whith the cone $C$ and the function $\theta$ evaluated at point $a_{M}$. Using the definition of $\theta$, one gives to this inclusion the form $0 \in \partial \psi_{D}\left(u_{F}\right)+u_{F}$, where $D$ denotes the set $T\left(a_{M}\right) \cap\left(C\left(a_{M}\right)+u_{L}\right)$. Through elementary Convex Analysis, this means that $u_{F}$ equals the proximal point to the origin in this set, with regard to the usual Euclidean metric of $\mathbb{R}^{3}$. For the traditional, isotropic, Coulomb law, $D$ is a disk, so the proximal point is specially easy to calculate.

Stage 3. Calculate $a_{F}=a_{M}+\frac{1}{2} h u_{F}$.

REMARK 16.1 Even if one assumes isotropic friction, computing the motion 
of systems involving three-dimensional friction is not in general as simple as above, due to the role of the mapping $G_{q}$. The determination of $u_{F}$, at each time-step, usually is a non convex problem, which may possess several solutions.

EXAMPLE 16.2 The numerical technique presented here has been applied [24] to the motion of a particle P submitted to gravity and confined by a plane boundary with prescribed motion. This particle represents an object which, in the case of contact, may slide on the boundary, without rolling nor tumbling. The plane boundary may be the ground surface, in the course of an earthquake, or also a vibrating table. Motions of the following sort are common in industrial conveyors.

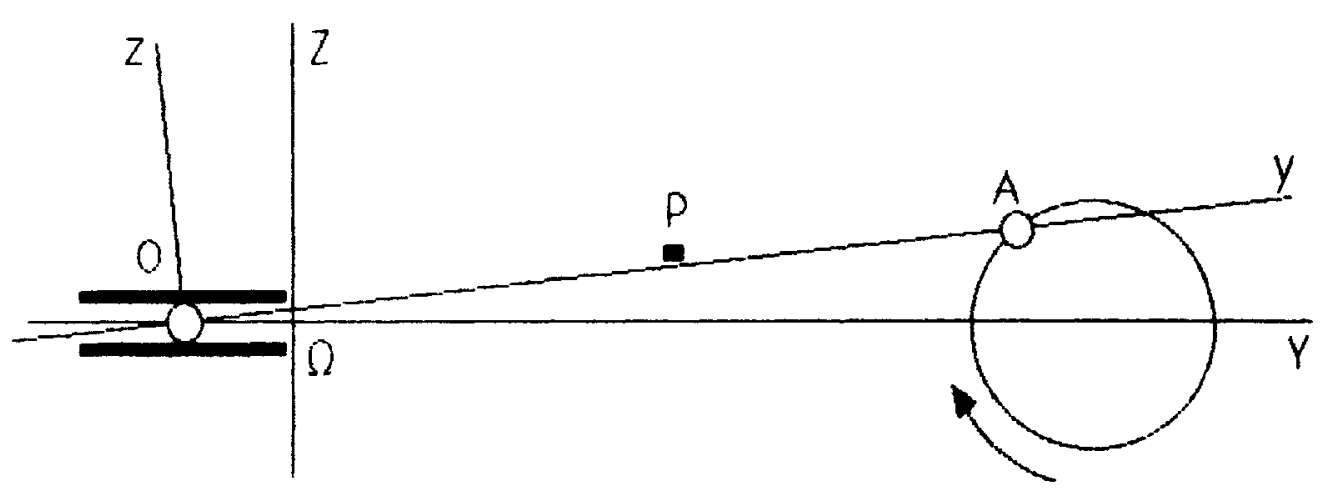

Figure 16.1

The vibrating table is assumed to have the motion of the shaft in a crank and shart mechanism. Let orthonormal axes Oxyz be attached to the table, with Oxy in its surface. Axes Oy and $O z$ move in flxed plane $\Omega Y z$. The point $O$ is guided along a segment of the line $\Omega Y$. The point $A(0, a, 0)$ of the table is astrained, by an eccentric, to describe, at constant velocity, a circle in the plane $\Omega Y Z$, centered on $\Omega Y$. Therefore, the plane $\Omega X Y$ is the mean position of the vibrating table. 
In order to produce a clear pattern of trajectories, the whole machine is set at a slant: the plane $\Omega Z X$ is vertical, but $\Omega Z$ is not in line with gravity, Hence, $\Omega X$ is the direction of steepest descent in the plane $\Omega X Y$ and determines the general trend of the motion of $P$.

A computer program, using the numerical technique described above, draws the projections to Oxy an to Oyz of the trajectories of $P$ relative to the table. No experiment has so far been conducted for comparison with reality.

At the initial instant, $P$ is left on the table with zero relative velocity. Subsequent trajectories are drawn for several choices of this initial position, at various distances of ox. Motions taking place sufficiently far from $O x$ involve intermittent contact break; the loops then observed on the Oyz projection correspond to the parabolic motion that $P$ have, when referred to fixed axes.

Here are the values of the system constants, understood as referring to c.g.s. units. Gravity equals $981 ; \Omega 2$ makes an angle of $13^{\circ}$ with the upward vertical direction. The eccentric has radius 0.5 and rotation speed 10 rps. The length $O A$ equals 50 . Friction coefficient is taken equal to 0.4 .

With this values, it turns out that the table shake is strong enough for leaving no place where $P$ could rest without sliding. The whole pattern of trajectories admits $O x$ as an axis of symmetry. Depending on the direction in which the eccentric rotates, this line is a locus of attraction or of repulsion, a fact which could be asserted from qualitative reasoning. More inexpected is the existence of other lines of attraction or of repulsion (they exchange their roles when rotation is reversed), parallel to this one. Such a "quantic" effect appears to be connected with the number of flappings that the table 
performs while the particle runs through each episode of contact-free motion. Actually, the farther they lie from $0 x$, the more confuse these attraction loci appear, due to the chaotic behaviour that trajectories then have.

Figure 16.2 shows the trajectories of $P$, consequent to 15 initial positions equally spaced from $y=5$ to $y=320$; the eccentric rotates in the reverse direction to that indicated on Fig. 16.1.

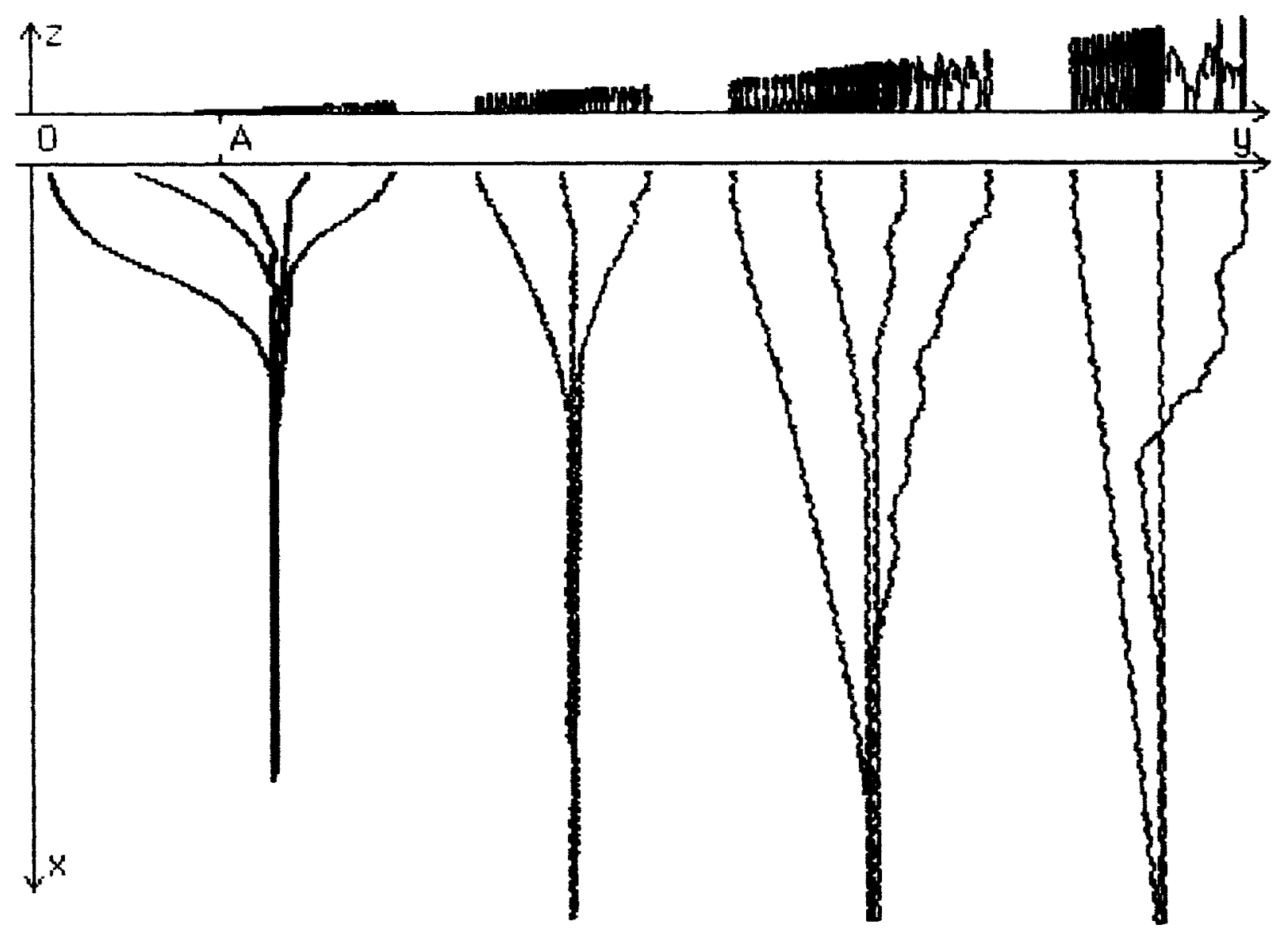

Figure 16.2

Fig.16.3 displays a larger scale drawing, corresponding to the same direction of rotation as on Fig. 16.1. The trajectories correspond to 7 initial positions equally spaced from $y=5$ to $y=150$. 


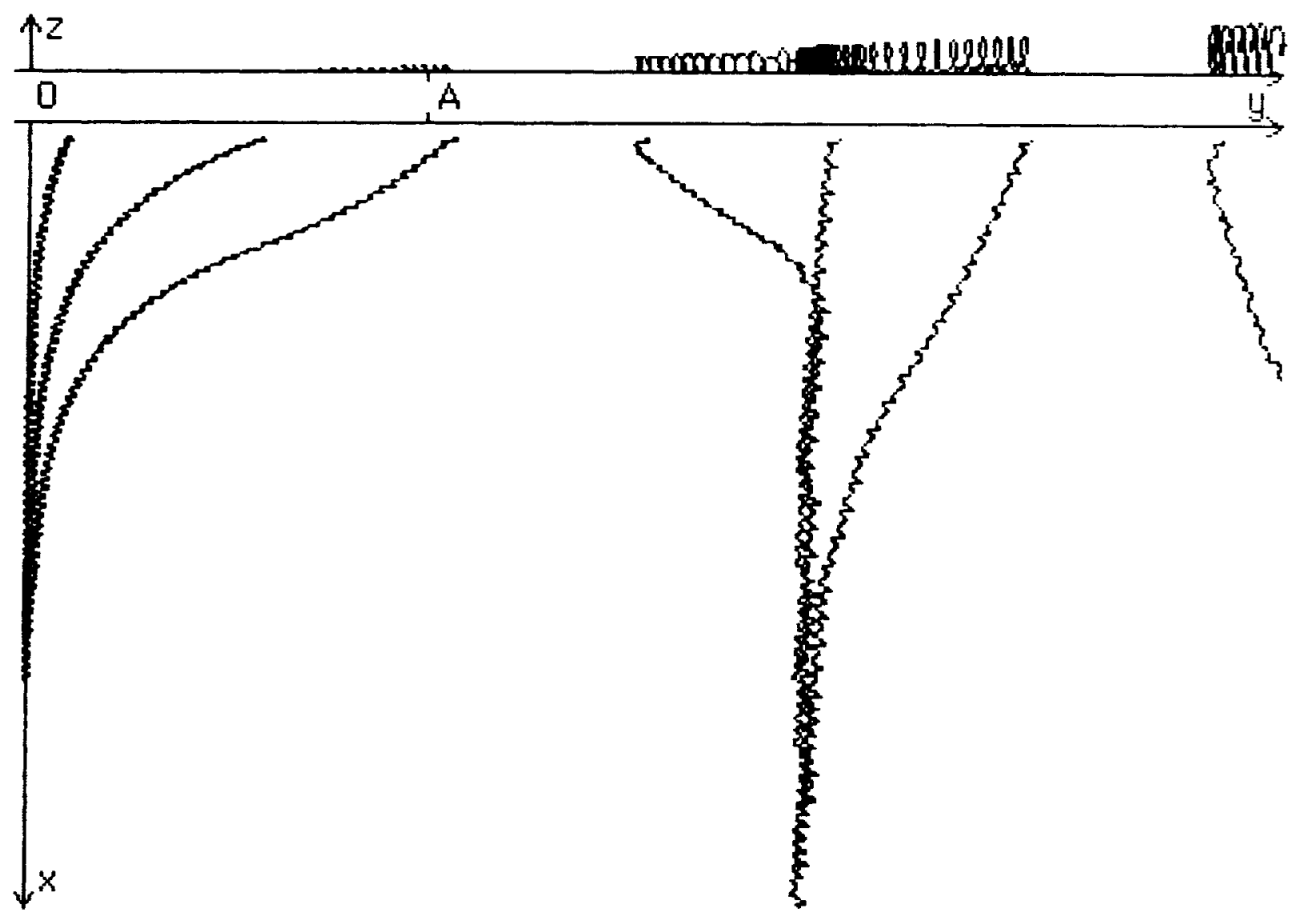

Figure 16.3

\section{STATIC AND DYNAMIC'FRICTIONS}

In many familiar situations, friction appears higher when the contacting bodies are to be set in motion from rest than during an episode of established sliding. Such an effect of "tangent sticking" is traditionally accounted for by introducing a larger value for the static friction coefficient, i.e. relative to zero sliding velocity, than for the dynamic one.

The numerical techniques proposed in the foregoing sections handle this refinement without difficulty. There only 15 , in each step of time discretization, to make the cone $C$ depend on the sliding status in the antecedent step. For instance, in the algorithm described in Sec.15, this 
antecedent step involves sliding or no sliding, according to $-H_{M}^{-1} U_{L}$ belonging to $C$ or not. This precisely makes one of the branching conditions one anyway had to consider.

At the stage of the analytical formulation, one has to make the cone $\mathcal{C}$ depend not only on $q$, but also on $\mathcal{U}$ or, if the possibllity of a velocity jump is considered, on $\mathcal{U}^{-}$. At first glance, this seems to reduce the advantage of the formulations of friction presented in the foregoing. But the example below tends to demonstrate that, far from being an heterogeneous addition to the previous theorization, such a refinement actually proves inherent in the subject matter. In fact, this example shows that, even if one starts from a law of friction with single coefficient, it may happen that the logical derivation of consequences eventually makes some contact force appear to obey a Coulomb law with coefficient depending on sliding velocity.

Let us consider again the situation of Sec.13, namely, in a position q of the system, with $f(q)=0$, the range $G_{q}\left(\mathbb{R}^{n}\right)$ is assumed to reduce to a two-dimensional subspace $\mathbb{W}$ of $\mathcal{E}_{3}$, different from the common tangent plane $T$ to the contacting bodies. In a motion with continuous velocity, it is assumed that the contact force $\boldsymbol{R} \in \mathcal{F}_{3}$ is related to $\boldsymbol{U}$ through Coulomb's law, expressed as before in the form

$$
-U \in \operatorname{proj}_{\mathcal{T}} \partial \psi_{C}(\mathcal{R})
$$

As already observed, $\mathcal{R}$ pertains to the equations of mechanics only through its equivalence class modulo $\operatorname{ker} G_{q}^{*}$, a natural representative of which is

$$
\mathcal{R}^{*}=\operatorname{proj}_{\mathcal{W}} \mathcal{R}
$$

Furthermore, since $\mathcal{U}$ essentially belongs to the tangent vector plane $T$, it has the form $U=s I$, where $I$ denotes a unit vector of the line $T \cap W$, and $s \in \mathbb{R}$. We are going to show that the resulting relation between $s$ and $R^{*}$ may 
have a more general form than it has been assumed in Sect.13.

Let us consider only, for simplicity, the traditional case where $c$ is a cone of revolution about $\mathcal{N}$. Clearly, for all $s>0$, the set of the values of $R$ that relation (17.1) associates with $U=s I$ consists of a certain boundary half-line, say $\mathcal{H}^{+}$, of the cone. With all $s<0$ is associated the half-line $\mathcal{H}^{-}$, symmetric to $\mathcal{H}^{+}$relative to the cone axis. Finally, to $s=0$, correspond all the points of $\mathcal{C}$. The orthogonal projections of $\mathcal{H}^{+}$and $\mathcal{H}^{-}$to $\mathcal{W}$ are two ilalf-lines, say $\mathcal{D}^{+}$and $\mathcal{D}^{-}$, lying on the same side of $T \cap W$. The expected relation between $s$ and $R^{*}$ is then expressed by the three implications

$$
\begin{aligned}
& \mathrm{s}>0 \Rightarrow \mathbb{R}^{*} \in \mathcal{D}^{+} \\
& \mathrm{s}<0 \Rightarrow \mathcal{R}^{*} \in \mathcal{D}^{-} \\
& \mathrm{s}=0 \Rightarrow \mathbb{R}^{*} \in \operatorname{proj}_{\mathcal{W}} C .
\end{aligned}
$$

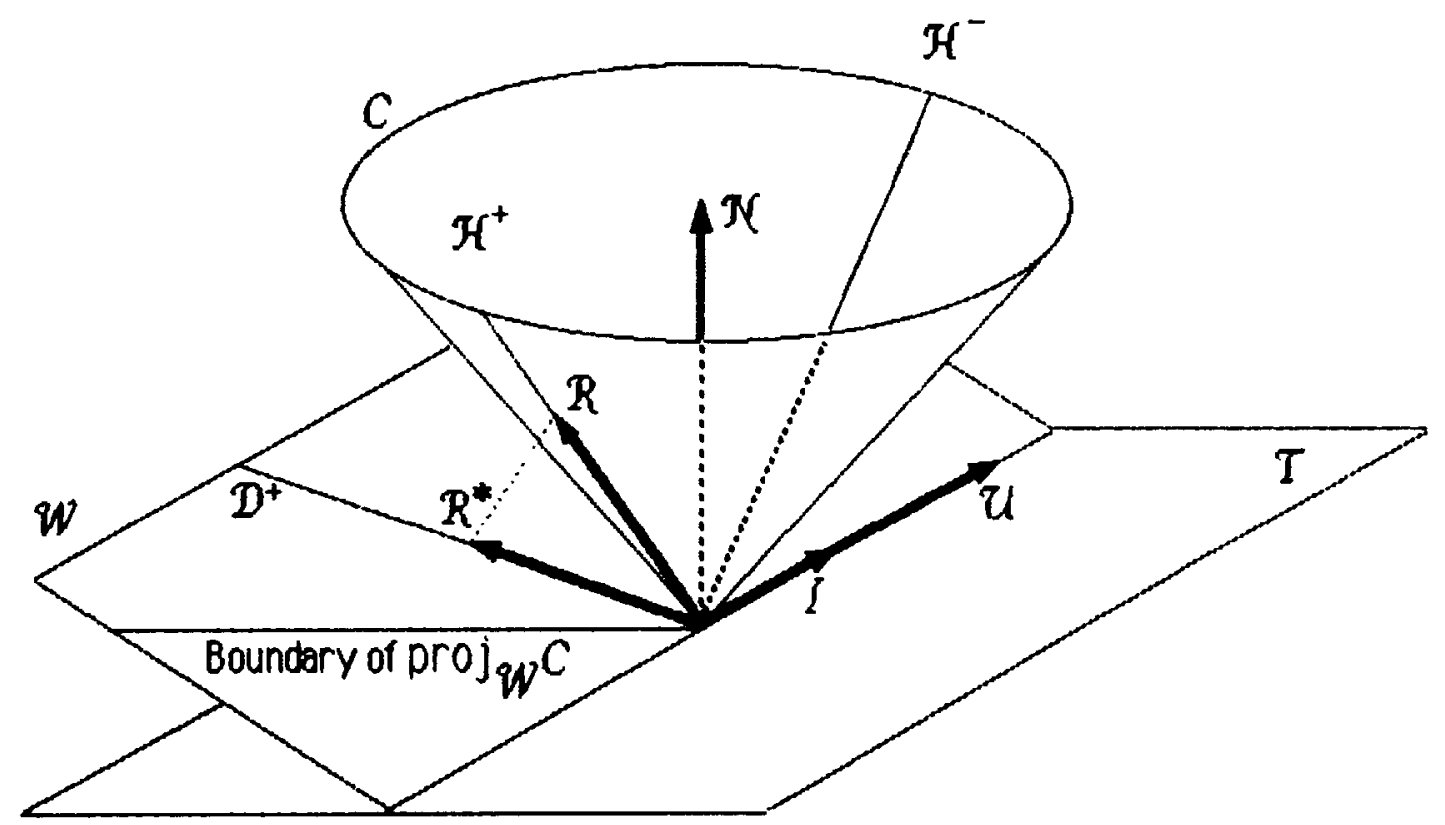

Figure 17.1

Depending on the span of the cone $C$ and on the angle that $W$ makes with $\mathcal{N}$, the orthogonal projection of $C$ to $\mathcal{W}$ may equal the whole of $\mathcal{W}$ or some 
angular region $C^{*}$ of this two-dimensional space.

In the latter case, $C^{*}$ contains $\mathfrak{D}^{+}$and $\mathcal{D}^{-}$but has no reason in general to possess them as its edges: this means that the relation found between $u$ and $\mathcal{R}^{*}$ is equivalent to a two-dimensional coulomb law with static coefricient larger than the dynamic one. The equality of these coefficients, namely the simple case studied in Sec. 13, is however achieved if $\boldsymbol{W}$ is orthogonal to $T$.

If $\operatorname{proj}_{\mathcal{W}} C=W$, every value of $\mathcal{R}^{*}$ in $W$ is associated with $U=0$, possibly making with $\mathcal{N}$ an angle larger than $\pi / 2$. In statical problems, this could be described as a wedging effect.

The above discussion provides an example of the interaction between the constraints of the system and the frictional effects at possible contact points. The treatment of systems involving several contacts with coulomb friction, a question left aside in these lectures, leads in general to simllar situations.

\section{REF́ERENCES}

1. Delassus, E.: Mémoire sur la théorie des liaisons finies unilatérales, Ann. Sci. Ecole Norm. Sup., 34 (1917), 95-179.

2. Pérès, J.: Mécanique Générale, Masson, Paris 1953.

3. Moreau, J.J.: Les llaisons unllatérales et le principe de Gauss, C.R. Acad. Sci. Paris, 256 (1963), 871-874.

4. Moreau, J.J.: Quadratic programming in mechanics: dynamics of one-sided constraints, SIAM J. Control, 4(1966), 153-158.

5. Dunford, N. and J.T. Schwartz: Linear Operators, Part I: General Theory, Interscience Pub. Inc., New York 1957.

6. Dinculeanu, N.: Vector Measures, Pergamon, London, New York 1967.

7. Moreau, J.J.: Bounded variation in time, in: Topics in Nonsmooth Mechanics (Ed. J.J. Moreau, P.D. Panagiotopoulos and G. Strang), Birkhäuser, to appear.

8. Pandit, S.G. and S.G. Deo: Differential Systems Involving Impulses, 
Lecture Notes in Math., vol. 954, Springer-Verlag, Berlin, Heidelberg, New York 1982.

9. Moreau, J.J.: Une formulation de la dynamique classique, C.R. Acad. Sci. Paris, Sér.11, 304 (1987), 191-194.

10. Panagiotopoulos, P.D.: Inequality Problems in Mechanics and Applications, Birkhäuser, Boston, Basel, Stuttgart 1985.

11. Monteiro Marques, M.D.P.: Chocs inélastiques standards: un résultat d'existence, Travaux du Séminaire d'Analyse Convexe, Univ. des Sci. et Techniques du Languedoc, vol. 15, exposé $n^{\circ}$ 4, Montpellier, 1985.

12. Monteiro Marques, M.D.P.: Rafle par un convexe semi-continu inférieurement, d'intérieur non vide, en dimension finie, C.R. Acad. Sci. Paris, Sér.l, 299 (1984), 307-310.

13. Monteiro Marques, M.D.P.: Inclusões Diferenciais e Choques Inelásticos, Doctoral Dissertation, Faculty of Sciences, University of Lisbon, 1988.

14. Moreau, J.J.: Liaisons unilatérales sans frottement et chocs inélastiques, C.R. Acad. Sci. Paris, Sér.11,296 (1983), 1473-1476.

15. Moreau, J.J.: Standard inelastic shocks and the dynamics of unilateral constraints, in: Unilateral Problems in Structural Analysis (Ed. G. Del Piero and F. Maceri), CISM Courses and Lectures No.288, Springer-Verlag, Wien, New York 1985.

16. Schatzman, M.: A class of nonlinear differential equations of second order in time, J. Nonlinear Analysis, Theory, Methods and Appl.,2 (1978),355-373.

17. Buttazzo, $G$. and D. Percivale: On the approximation of the elastic bounce problem on Riemannian manifolds, J. Diff. Equations, 47 (1983), 227-245.

18. Carriero, $M$. and $E$. Pascali: Uniqueness of the one-dimensional bounce problem as a generic property in $L^{\prime}([0, T] ; R)$, Bolletino U.M.I.(6) $1-A$ (1982), 87-91.

19. Percivale, D.: Uniqueness in the elastic bounce problem, J. Diff. Equations, 56(1985), 206-215.

20. Moreau, J.J.: Sur les lois de frottement, de plasticité et de viscosité, C.R. Acad. Sci. Paris, Sér.A, 271 (1970), 608-611.

21. Moreau, J.J: On unllateral constraints, friction and plasticity, in: New Variational Techniques in Mathematical Physics (Ed. G. Capriz and G. Stampacchia), CIME 2 ciclo 1973, Edizioni Cremonese, Roma, 1974, 173-322.

22. Moreau, J.J.: Application of convex analysis to some problems of dry friction, in: Trends in Applications of Pure Mathematics to Mechanics, 
vol.2 (Ed. H. Zorski), Pitman Pub. Ltd., London 1979, 263-280.

23. Duvaut, G. and J.L. Lions: Les Inéquations en Mécanique et en Physique, Dunod, Paris 1972.

24. Moreau, J.J.: Dynamique de systèmes à liaisons unilatérales avec frottement sec éventuel; essais numériques, Note Technique 85-I, Lab. de Mécanique Générale des Milieux Contínus, Univ. des Sci. et Techniques du Languedoc, Montpellier, 1985.

25. Moreau, J.J.: Une formulation du contact à frottement sec; application au calcul numérique, C.R. Acad. Sci. Paris, Sér.11,302 (1986), 799-801.

26. Lecornu, L.: Sur la loi de Coulomb, C.R. Acad. Sci. París, 140 (1905), 847-848.

27. Oden, J.T. and J.A.C. Martins: Models and computational methods for dynamic friction phenomena, Computer Methods in Appl. Mech. and Engng., 52 (1985), 527-634.

28. Jean, $M$. and $G$. Touzot: Implementation of unilateral contact and dry friction in computer codes dealing with large deformations problems, to appear in: Numerical Methods in Mechanics of Contacts Involving Friction, J. de Mécanique théor. et appl., Special issue, 1988.

29. Abadie, J.: On the Kuhn-Tucker theorem, in: Nonlinear Programming (Ed. J.Abadie), North-Holland Pub. Co., Amsterdam 1967, 19-36.

30. Jean, M. and J.J. Moreau: Dynamics in the presence of unilateral contacts and dry friction; a numerical approach, in: Unllateral Problems in Structural Analysis 2" (Ed. G. Del Piero and F. Maceri), CISM Courses and Lectures No 304, Springer-Verlag, Wien 1987, 151-196.

31. Moreau, J.J.: Evolution problem associated with a moving convex set in a Hilbert space, J. Diff. Equations, 26 (1977), 347-374.

32. Moreau, J.J.: Sur les mesures différentielles de fonctions vectorielles et certains problèmes d'évolution, C.R. Acad. Sci. Paris, Sér.A, 282 (1976), 837-840.

33. Jean, M. and E. Pratt: A system of rigid bodies with dry friction, Int. J. Engng. Sci., 23 (1985), 497-513.

34. Rockafellar, R.T.: Convex Analysis, Princeton Univ. Press, Princeton 1970.

35. Moreau, J.J.: Fonctionnelles Convexes, Lecture Notes, Séminaire sur les Equations aux Dérivées Partielles, Collège de France, Paris 1967.

36. Painlevé, P.: Sur les lois du frottement de glissement, C.R. Acad. Sci. Paris, 121 (1895), 112-115. Same title, ibld. 141 (1905), 401-405 and 141 (1905), 546-552.

37. Delassus, E.: Considérations sur le frottement de glissement, Nouv. Ann. 
de Math. (4ème série), 20 (1920), 485-496.

38. Delassus, E.: Sur les lois du frottement de glissement, Bull. Soc. Math. France, 51 (1923), 22-33.

39. Klein, F.: Zu Paínlevés Kritik des Coulombschen Reibungsgesetze, Zeitsch. Math. Phys., 58 (1910), 186-191.

40. Mises, R.v.: Zur Kritik der Reibungsgesetze, ibid., 191-195.

41. Hamel, G.: Bemerkungen zu den vorstehenden Aufsätzen der Herren F. Klein und R. v. Mises, ibid., 195-196.

42. PrandtI, L.: Bemerkungen zu den Aufsätzen der Herren F. Klein, R. v. Mises und G. Hamel, ibid., 196-197.

43. Beghin, $H$.: Sur certains problemes de frottement, Nouv. Ann. de Math., 2 (1923-24), 305-312.

44. Beghin, $H .:$ Sur l'indétermination de certains problèmes de frottement, Nouv. Ann. de Math., 3 (1924-25), 343-347. 